\title{
Particle Structure Analysis of Soliton Sectors in Massive Lattice Field Theories
}

\author{
P. A. Marchetti
}

Dipartimento di Fisica dell'Universitá di Padova, Via Marzolo 8, I-35131 Padova, Italy

\begin{abstract}
We discuss the particle structure in the soliton sectors of massive lattice field theories by means of convergent cluster expansions. In several models we prove that the soliton field operator with lowest charge couples the vacuum to a stable one-particle state, in a suitable region of the coupling parameter space. Both local and stringlike solitons are analyzed. We also show that the mass of the local soliton equals the surface tension.
\end{abstract}

\section{Introduction}

A procedure to construct quantum solitons in lattice field theories has been proposed in paper I $(=[1])$. The basic idea is to apply an Osterwalder-Schrader (O.S.) reconstruction theorem [2] to mixed order-disorder correlation functions.

We now shortly describe how mixed order-disorder correlation functions are constructed and how they are related to solitons.

The expectation value of a disorder field, $D(\omega)$, is given by

$$
\langle D(\omega)\rangle \equiv \lim _{\Lambda \uparrow \mathbf{z}_{1 / 2}^{d}} \frac{Z_{\Lambda}(\omega)}{Z_{\Lambda}},
$$

where $\omega$ is an external hyper-gauge field with values in a discrete abelian group $\mathscr{Z}$; $Z_{\Lambda}$ is the partition function of the theory in a lattice $\Lambda \subset \mathbf{Z}_{1 / 2}^{d}$ and $Z_{\Lambda}(\omega)$ denotes the partition function of the model coupled to the external field $\omega$.

The prefix hyper-means that $\omega$ has a rank ${ }^{1}$ higher than the ranks of the basic fields of the theory. For example, in the models discussed here, $\omega$ has rank 1 in the scalar and fermion theories (i.e. it is a true lattice gauge field) and rank 2 in gauge theories.

Assume that $\langle D(\omega)\rangle$ depends on the curvature, $d \omega$, of $\omega$ and the support of $(d \omega)^{*}$, the dual of $d \omega$, is given by a finite set of points $\left\{x_{i}\right\}_{i=1}^{n}$ with $(d \omega)^{*}\left(x_{i}\right)=q_{i}$, then $\langle D(\omega)\rangle$ will be denoted by $\left\langle D\left(x_{1}, q_{1}, \ldots, x_{n}, q_{n}\right)\right\rangle$.

\footnotetext{
${ }^{1}$ A lattice field of rank $k$ with values in a space $W$ is a map from $k$-dimensional cells of the lattice to W
} 
A function of the basic fields of the theory with support on a compact, connected set of cells $\Gamma$ (such as the Wilson loop in gauge theories) will be denoted by $\mathcal{O}(\Gamma)$. The correlation functions to which the reconstruction theorem applies are then given by

$$
S_{n, m}\left(x_{1}, q_{1}, \ldots, x_{n}, q_{n}, \Gamma_{1}, \ldots, \Gamma_{m}\right) \equiv\left\langle D\left(x_{1}, q_{1}, \ldots, x_{n}, q_{n}\right) \mathcal{O}\left(\Gamma_{1}\right) \ldots \mathcal{O}\left(\Gamma_{m}\right)\right\rangle
$$

for $\sum_{i} q_{i}=0$, and correlation functions with non-vanishing total charge are defined by a limiting procedure, i.e. by sending a charge to infinity.

$$
\begin{aligned}
\text { More precisely for } \sum_{i=1}^{n} q_{i} & =q, \\
S_{n, m}\left(x_{1}, q_{1}, \ldots, x_{n}, q_{n} ; \Gamma_{1} \ldots \Gamma_{m}\right) & =\lim _{x \rightarrow \infty} c_{q} S_{n+1, m}\left(x_{1}, q_{1}, \ldots, x_{n}, q_{n}, x, q, \Gamma_{1} \ldots \Gamma_{m}\right),
\end{aligned}
$$

where $c_{q}$ is a normalization constant needed if $\lim S_{2}(0,-q, x, q) \neq 0$ and then given by

$$
c_{q}=\left[\lim _{x \rightarrow \infty} S_{2}(0,-q, x, q)\right]^{-1 / 2} .
$$

The construction of the soliton sectors is obtained by means of the following

Theorem 1.1. [I] If the set of correlation functions $\left\{S_{n, m}\right\}$ is

i) lattice translation invariant;

ii) O.S. (reflection) positive;

iii) satisfies cluster properties; $\mathscr{H}$ then one can reconstruct from $\left\{S_{n, m}\right\}$

a) a separable Hilbert space, $\mathscr{H}$, of physical states;

b) a vector $\Omega \in \mathscr{H}$ of unit norm, the vacuum;

c) a selfadjoint transfer matrix $T$ with norm $\|T\| \leqq 1$ and unitary spatial translation operators $U_{\mu}, \mu=1 \ldots d-1$, such that

$$
T \Omega=U_{\mu} \Omega=\Omega
$$

d) $\Omega$ is the unique vector in $\mathscr{H}$ invariant under $T$ and $U$.

If moreover the limits (1.3) vanish, then $\mathscr{H}$ splits into orthogonal sectors $\mathscr{H}_{q}$, $q \in \mathscr{Z}$, i.e.

$$
\mathscr{H}=\bigoplus_{q} \mathscr{H}_{q}
$$

and the sectors $\mathscr{H}_{q}, q \neq 0$ are (lattice) soliton sectors.

In the reconstruction theorem a set of states, $\left|x_{1}, q_{1}, \ldots, x_{n}, q_{n}, \Gamma_{1}, \ldots, \Gamma_{m}\right\rangle \in \mathscr{H}$, naturally arises, corresponding to monomials of order and disorder fields with $x_{i}$ and $\Gamma_{j}$, in the positive time lattice. We denote by $F_{+}$the corresponding linear span. On $F_{+}$the scalar product is defined by

$$
\begin{aligned}
& \left\langle x_{1}, q_{1}, \ldots, x_{n}, q_{n}, \Gamma_{1}, \ldots, \Gamma_{m} \mid y_{1}, q_{1}^{\prime}, \ldots, y_{a}, q_{a}^{\prime}, \Gamma_{1}^{\prime}, \ldots, \Gamma_{b}^{\prime}\right\rangle \\
& \left.\quad=<D\left(r x_{1},-q_{1}, \ldots, r x_{n},-q_{n}, y_{1}, q_{1}^{\prime}, \ldots, y_{a}, q_{a}^{\prime}\right) \prod_{j=1}^{m} \theta \mathcal{O}\left(\Gamma_{j}\right) \prod_{i=1}^{b} \mathcal{O}\left(\Gamma_{i}^{\prime}\right)\right\rangle \\
& \quad \equiv S_{n+a, m+b}\left(r x_{1},-q_{1}, \ldots, y_{a}, q_{r}^{\prime}, \theta \Gamma_{1}, \ldots, \theta \Gamma_{m}, \Gamma_{1}^{\prime}, \ldots, \Gamma_{b}^{\prime}\right),
\end{aligned}
$$

where $r$ denotes the reflection in the time zero plane and $\theta$ denotes the involution of the O.S. reconstruction theorem. (The symbol $\langle\cdot, \cdot\rangle$ is also sometimes used for the scalar product in $\mathscr{H}$.) 
Field operators $A\left(x_{1}, q_{1}, \ldots, x_{n}, q_{n}, \Gamma_{1}, \ldots, \Gamma_{m}\right)$ with $x_{i}$ and $\Gamma_{j}$ contained in the time strip $[0, t], t \in \mathbf{Z}_{+}$, naturally act on $T(t) F_{+}$by

$$
\begin{aligned}
& A\left(x_{1}, q_{1}, \ldots, x_{n}, q_{n}, \Gamma_{1}, \ldots, \Gamma_{m}\right) T(t)\left|x_{1}^{\prime}, q_{1}^{\prime}, \ldots, x_{a}^{\prime}, q_{a}^{\prime}, \Gamma_{1}^{\prime}, \ldots, \Gamma_{b}^{\prime}\right\rangle \\
& \quad=\left|x_{1}, q_{1}, \ldots, x_{n}, q_{n},\left(x_{1}^{\prime}\right)_{t}, q_{1}^{\prime}, \ldots,\left(x_{a}^{\prime}\right)_{t}, q_{a}^{\prime} ; \Gamma_{1}, \ldots, \Gamma_{m},\left(\Gamma_{1}^{\prime}\right)_{t}, \ldots,\left(\Gamma_{b}^{\prime}\right)_{t}\right\rangle,
\end{aligned}
$$

where $(\cdot)_{t}$ denotes translation by $t$ in the time direction.

In particular $A(\Gamma)$ is called an order field operator and

$$
A(x, q) \equiv s_{q}(x)
$$

is called a soliton field operator of charge $q$.

To be precise, we define a sector $\mathscr{H}_{q}$ of $\mathscr{H}$ to be a (lattice) soliton sector if 1$)$ it is invariant under the action of $T, U_{\mu}$ and of the set of field operators $\left\{A\left(\Gamma_{1} \ldots \Gamma_{m}\right)\right\}_{m=0}^{\infty}$; 2) it does not contain any lattice translation invariant vector, 3 ) the labelling charge $q$ is a "topological charge," in the jargon of quantum field theory, or a "defect charge," in the jargon of statistical mechanics (for more details see I).

All the above considerations are made under the assumption that $\langle D(\omega)\rangle$ depends only on $d \omega$. However the whole structure we have discussed can be easily generalized to the case of a full $\omega$-dependence. In particular Theorem 1.1 holds provided a suitable definition of order-disorder correlation functions is made (see I and, here, Sect. 2.2).

A particle analysis of the (lattice) soliton sectors constructed as above can be obtained using the excitation expansions [3].

The correlation functions $\left\{S_{n, m}\right\}$ admit a representation in terms of line defects, $v$, carrying a charge with values in the discrete abelian group $\mathscr{Z}$ :

$$
\begin{aligned}
& S_{n, m}\left(x_{1}, q_{1}, \ldots, x_{n}, q_{n}, \Gamma_{1} \ldots \Gamma_{m}\right) \\
& =\lim _{\Lambda \uparrow \mathbf{Z}_{1 / 2}} \frac{\sum_{v: \partial v=\left\{x_{1}, q_{1} \ldots x_{n}, q_{n}\right\}} Z_{\Lambda}^{\left\{\Gamma_{1} \ldots \Gamma_{m}\right\}}(v)}{\sum_{v: \partial v=0} Z_{\Lambda}(v)},
\end{aligned}
$$

where $Z_{\Lambda}^{\left\{\Gamma_{1} \ldots \Gamma_{m}\right\}}(v)$ is the statistical weight for the defect configuration $v$ in the lattice $\Lambda$ in the presence of $\mathcal{O}\left(\Gamma_{1}\right) \ldots \mathcal{O}\left(\Gamma_{n}\right), Z_{A}^{\{\phi\}} \equiv Z_{A}$ and $\partial v$ denotes the boundary of the charged defects.

Applied to the two-point function of the soliton field operator with minimal charge, Eq. (1.7) expresses $S_{2}(x,-1, y, 1) \equiv\left\langle s_{1}(x) \Omega, s_{1}(y) \Omega\right\rangle$ in terms of a sum over the configurations of a fluctuating line defect joining $x$ to $y$.

For theories involving continuous fields, this representation in terms of fluctuating lines can be rigorously analyzed, in the region of coupling constants for which (massive) soliton sectors exist, by means of a combined low and high temperature (C.L.H.T.) expansions, similar in spirit (but of course much easier) to the G.J.S. expansion [4] for $\phi_{2}^{4}$ in the broken symmetry phase.

An excitation analysis of the cluster expansion for $S_{2}(x,-1, y, 1)$, typically allows to prove a decay law

$$
\sum_{\vec{x}} S_{2}(0,-1, x, 1) \equiv \sum_{\vec{x}}\left\langle s_{1}(0, \overrightarrow{0}) \Omega, T(t) s_{1}(0, \vec{x}) \Omega\right\rangle \underset{t \uparrow \infty}{\sim} e^{-m t}\left(1+e^{-\mu t}\right)
$$

with $x=(t, \vec{x})$ and $m, \mu$ strictly positive constants. 
Such behaviour shows (see $[1,3])$ that the soliton field operator $s_{1}(x)$ couples the vacuum to a stable massive one-particle state.

In this paper we also prove that the soliton mass $m$ in (1.8), equals the surface tension $\tau_{1}$ in the models in which $\langle D(\omega)\rangle$ depends on $d \omega$. by

In a theory with $\mathscr{Z}$-valued line defects, the surface tension $\tau_{q}, q \in \mathscr{Z}$, is defined

$$
\tau_{q}=\lim _{\Lambda \uparrow \mathbf{Z}_{1 / 2}^{d}}-\frac{1}{T} \ln \frac{Z_{\Lambda}^{(q)}}{Z_{\Lambda}} .
$$

In (1.9) $\Lambda$ is a finite lattice centered at the origin with sides of length $L+1$ in the space directions and $T+1$ in the direction, $Z_{A}(q)$ is the partition function obtained from $Z_{A}$ by modifying the b.c. in such a way as to introduce a line defect of charge $q$ joining $(T, \overrightarrow{0})$ to $(-T, \overrightarrow{0})$.

The equation

$$
\tau_{1}=m
$$

was conjectured for $\phi_{2}^{4}$ in the continuum in [5], where the inequality $m \leqq \tau_{1}$ was established for $\phi_{2}^{4}$.

In the models we consider, we prove (1.10) using the excitation expansion, as suggested by Fröhlich [6].

The paper is organized as follows:

In Sect. 2 the lattice field theories we analyze are introduced, correlation functions (1.2) are defined and the main results are stated.

In Sect. 3 we construct the C.L.H.T. expansion and prove clustering for the models with $\langle D(\omega)\rangle$ depending on $d \omega$; via Theorem 1.1 one can then construct the soliton sectors.

In Sect. 4 we prove the behaviour (1.8) and in Sect. 5 the equality (1.10) for such models.

In Sect. 6 clustering and particle structure analysis are discussed for the models with $\langle D(\omega)\rangle$ depending on the full $\omega$.

Basic definitions and notations for lattice field theories are as in I.

\section{Lattice Field Theories, Disorder Fields and Solitons}

\subsection{Models with Local Soliton Sectors}

As discussed in I, lattice field theories which possess soliton sectors are:

- in $d=2$ scalar or fermion theories with spontaneous symmetry breaking of a global discrete abelian group $\mathscr{Z}$;

- in $d=3$ gauge theories with matter fields in the superconducting phase, in which a discrete abelian subgroup, $\mathscr{Z}$, of the gauge group remains unbroken.

As examples we consider here

a) $\phi^{4}$ in $d=2$,

b) non compact ( $U(1)$ Higgs) model in $d=3$,

c) $S U(N)$ gauge theory coupled to a matter (Higgs) field in the adjoint representation of $S U(N)$ in $d=3$ (for examples with fermions see I). 
The action in a finite lattice $\Lambda$, will be denoted by $S_{\Lambda}=S_{0 \Lambda}+S_{1 \Lambda}$, all parameters introduced are real positive.

a) $-\phi_{2}^{4}$

$$
\begin{gathered}
S_{0 \Lambda}=\frac{\beta_{H}}{2} \sum_{\langle x y\rangle \in \Lambda}\left(\phi_{x}-\phi_{y}\right)^{2}, \\
S_{1 \Lambda}=\lambda \sum_{x \in \Lambda}\left(\phi_{x}^{2}-1\right)^{2}
\end{gathered}
$$

with $\phi_{x} \in \mathbf{R}$

- $S_{1 \Lambda}$ is invariant under the (hyper)gauge transformation

$$
\phi_{x} \rightarrow \exp \left(i \pi \sigma_{x}\right) \phi_{x}, \quad \sigma_{x} \in \mathscr{Z}=\mathbf{Z}_{2} \cong\{0,1\}
$$

- $S_{1 \Lambda}$ is invariant under the corresponding global transformation; such symmetry is spontaneously broken for large $\beta_{H}$ and $\lambda$.

b) $-(U(1) \text { Higgs })_{3}$,

$$
\begin{gathered}
S_{0 \Lambda}=\frac{\beta_{G}}{2} \sum_{p \in \Lambda}\left(d A_{p}\right)^{2} \\
S_{1 \Lambda}=\frac{\beta_{H}}{2} \sum_{\langle x y\rangle \in \Lambda}\left|\phi_{x}-e^{i A\langle x y\rangle} \phi_{y}\right|^{2}+\lambda \sum_{x \in A}\left(\left|\phi_{x}\right|^{2}-1\right)^{2}
\end{gathered}
$$

with $\phi_{x} \in \mathbf{C}, A_{\langle x y\rangle} \in \mathbf{R}$

- a gauge fixing term such as

$$
S_{f i x \Lambda}=\frac{1}{2} \sum_{x \in \Lambda}(\delta A)_{x}^{2}
$$

is added to the action and 0-b.c. on $A$ are imposed on $\partial \Lambda$.

- $S_{1 A}$ is invariant under the hypergauge transformation

$$
A_{\langle x y\rangle} \rightarrow A_{\langle x y\rangle}+2 \pi \sigma_{\langle x y\rangle}, \quad \sigma_{\langle x y\rangle} \in \mathscr{Z}=\mathbf{Z}
$$

- the model is in the superconducting phase for $\beta_{G}, \beta_{H}, \lambda$ large enough; the group of gauge transformations given by (2.2) with $\sigma_{\langle x y\rangle}=\sigma_{x}-\sigma_{y}, \sigma_{x} \in \mathbf{Z}$ is left unbroken.

c) $-(S U(N) \text { Higgs })_{3}$

$$
\begin{gathered}
S_{0 \Lambda}=\frac{\beta_{G}}{2} \sum_{p \in \Lambda}\left(1-\operatorname{Re} \chi\left(g_{\partial p}\right)\right), \\
S_{1 \Lambda}=\beta_{H} \sum_{\langle x y\rangle \in \Lambda}\left[1-\operatorname{Re}\left(\phi_{x}, U_{H}\left(g_{\langle x y\rangle}\right) \phi_{y}\right)\right]+\lambda \sum_{x \in \Lambda}\left(\left(\phi_{x}, \phi_{x}\right)-1\right)^{2},
\end{gathered}
$$

where $g_{\langle x y\rangle} \in S U(N), \chi$ is a faithful character of $S U(N), \phi_{x} \in V_{H}$, a vector space with inner product $(\cdot, \cdot)$ carrying a faithful representation $U_{H}$ of $S U(N) / \mathbf{Z}_{N}$.

- "1"-b.c. are imposed on $g$

- $S_{1 \Lambda}$ is invariant under the hypergauge transformations

$$
g_{\langle x y\rangle} \rightarrow g_{\langle x y\rangle} \xi\left(\sigma_{\langle x y\rangle}\right), \quad \sigma_{\langle x y\rangle} \in \mathscr{Z}=\mathbf{Z}_{N} \cong\{0, \ldots, N-1\},
$$

where $\xi$ is the representation of $\mathbf{Z}_{N}$ on $\mathrm{SU}(\mathrm{N})$

- the model is in the superconducting phase for $\beta_{G}, \beta_{H}, \lambda$ large enough, with the group of gauge transformations given by (2.3) with $\sigma_{\langle x y\rangle}=\sigma_{x}-\sigma_{y}, \sigma_{x} \in \mathbf{Z}_{N}$ left unbroken. 
To define the disorder field we introduce a $\mathscr{Z}$-valued hypergauge field $\omega$ of compact support and we couple it to the model, so that the action of the coupled model, $S_{0 \Lambda}(\omega)+S_{1 \Lambda}$, is hypergauge invariant. Explicitly we substitute:
a)
b)
c)

$$
\begin{gathered}
\phi_{x}-\phi_{y} \rightarrow \phi_{x}-e^{i \pi \omega\langle x y\rangle} \phi_{y}, \quad \omega_{\langle x y\rangle} \in \mathbf{Z}_{2}, \\
(d A)_{p} \rightarrow d A_{p}+2 \pi \omega_{p}, \quad \omega_{p} \in \mathbf{Z}, \\
\chi\left(g_{\partial p}\right) \rightarrow \chi\left(g_{\partial p} \xi\left(\omega_{p}\right)\right), \quad \omega_{p} \in \mathbf{Z}_{N} .
\end{gathered}
$$

The disorder field is then defined by

$$
D(\omega)=e^{-\left(S_{0}(\omega)-S_{0}\right)} \equiv \lim _{\Lambda \uparrow \mathbf{Z}_{1 / 2}^{d}} e^{-\left(S_{0 \Lambda}(\omega)-S_{0 \Lambda}\right)} .
$$

From the invariance of $S_{0 \Lambda}(\omega)+S_{14}$ under the hypergauge transformations (2. 1-3) it follows that $\langle D(\omega)\rangle$ depends only on $d \omega$ (at least if the finite lattice $\Lambda$ is convex). Moreover, since $d \omega$ is a field with support in $d$-dimensional cells, it follows that $(d \omega)^{*}$ has support on a set of points $\left\{x_{i}\right\}_{i=1}^{n}$, in the dual lattice, as required in (1.2).

The functions $\mathcal{O}(\Gamma)$ used in the mixed correlation functions (1.2) are hypergauge invariant but in general $\omega$-dependent and in this case they are denoted more precisely by $\mathcal{O}(\omega, \Gamma)$. In our models we take

a)

b)

c)

$$
\begin{gathered}
\mathcal{O}\left(\omega, \Gamma_{x y}\right)=\phi_{x} \prod_{b \in \Gamma_{x y}} e^{i \pi \omega_{b}} \phi_{y}, \\
\mathcal{O}(C)=\prod_{b \in C} e^{i A_{b}}, \quad \mathcal{O}\left(\Gamma_{x y}\right)=\phi_{x} \prod_{b \in \Gamma_{x y}} e^{i A_{b}} \phi_{y}, \\
\mathcal{O}(C)=\tilde{\chi}\left(\prod_{b \in C} g_{b}\right), \quad \mathcal{O}\left(\Gamma_{x y}\right)=\phi_{x} \prod_{b \in \Gamma_{x y}} U_{H}\left(g_{b}\right) \phi_{y},
\end{gathered}
$$

where $\Gamma_{x y}$ is a line from $x$ to $y, C$ a loop and $\tilde{\chi}$ a faithful character of $S U(N) / \mathbf{Z}_{N}$.

The soliton field operators $s_{q}(x)$ reconstructed from the correlation functions (1.2) are local and we call the corresponding superselection sectors local soliton sectors.

We collect our results in

Theorem 2.1. The models

- a) for $\beta_{H}$ large, $\beta_{H} / \lambda^{1 / 2}$ small, $\beta_{H} \gg\left|\ln \left(\beta_{H} / \lambda\right)\right|$,

- b) c) for $\beta_{G}$ large, $\beta_{G} / \beta_{H}^{1 / 2}, \beta_{H} / \lambda$ small, $\beta_{G} \gg\left\{\left|\ln \left(\beta_{G} / \beta_{H}\right)\right|,\left|\ln \left(\beta_{H} / \lambda\right)\right|\right\}$

posses soliton sectors $\mathscr{H}_{q}$ labelled by the non-trivial elements $q$ of the group $\mathscr{Z}$ $\left.\left.\left.(=a) \mathbf{Z}_{2}, b\right) \mathbf{Z}, c\right) \mathbf{Z}_{N}\right)$.

Theorem 2.2. The soliton field operator $s_{1}(x)$ couples the vacuum to a stable oneparticle state, in particular the decay (1.8) holds with

a)

$$
m=2 \beta_{H}+\mathcal{O}\left(\beta_{H} / \lambda^{1 / 2}\right), \quad \mu=\mathcal{O}\left(\left|\ln \beta_{H} / \lambda\right|\right),
$$

b)

$$
m=2 \pi^{2} \beta_{G}+\mathcal{O}\left(\frac{\beta_{G}}{\beta_{H}^{1 / 2}}\right), \quad \mu=\min \left[\mathcal{O}\left(\left|\ln \beta_{G} / \beta_{H}\right|\right), \mathcal{O}(|\ln \beta / \lambda|)\right],
$$

c)

$$
m=\beta_{G}\left(1-\cos \frac{2 \pi}{N}\right)+\mathcal{O}\left(\frac{\beta_{G}}{\beta_{H}^{1 / 2}}\right), \quad \mu=\min \left[\mathcal{O}\left(\left|\ln \beta_{G} / \beta_{H}\right|\right), \mathcal{O}(|\ln \beta / \lambda|)\right] .
$$




\subsection{Models with Stringlike Solitons}

If we perturb the models described in the previous section by adding a new term in the action, $\beta_{B} S_{B A}$, which is not invariant under the $\mathscr{Z}$-valued hypergauge transformations, a different class of soliton sectors occurs, if the theory with $\beta_{B} \neq 0$ is still in the same phase in which the $\beta_{B}=0$ theory possesses local soliton sectors.

In $d=3$ such a situation occurs if we add to a gauge theory with matter fields in the superconducting phase, with $\mathscr{Z}$ unbroken, an interaction between the gauge field and a matter field which transform non-trivially under $\mathscr{Z}$.

As an example we consider the non-compact abelian Higgs model coupled to a fermion of fractional charge.

The fermion is coupled to the gauge field by a term in the action given by

$$
\beta_{B} S_{B 1 \Lambda}(\bar{\psi}, \psi, A)=\frac{\beta_{B}}{2} \sum_{\langle x y\rangle \in \Lambda} \bar{\psi}_{x}\left(1+\gamma_{\langle x y\rangle}\right) e^{\frac{i}{N} A\langle x y\rangle} \psi_{y},
$$

where $1<N \in \mathbf{Z}_{+}$, and we add a fermion mass term

$$
S_{1 \Lambda}(\bar{\psi}, \psi)=\sum_{x \in \Lambda} \bar{\psi}_{x} \psi_{x} .
$$

This model is of some interest in physics since it can be used to make an approximate description of the interaction, in a superconductor, between photons, electrons and the superconductor condensate, described by the Higgs field $\phi$.

Since the condensate has twice the charge of the electron, in this situation $N=2$.

In a finite lattice $\Lambda$, the disorder field is still defined by

$$
D(\omega)=e^{-\left(S_{0 \Lambda}(\omega)-S_{0 \Lambda}\right)} .
$$

However, since $S_{B A}$ not hypergauge invariant $\langle D(\omega)\rangle_{A}$ now depends on the full hypergauge field $\omega$.

As discussed in I, one must choose the support of $\omega$ in a suitable way in order to ensure O.S. positivity of the correlation functions, which is a necessary condition to obtain positivity of the space of states $\mathscr{H}$ in the reconstruction theorem.

Suppose $\operatorname{supp}(d \omega)^{*}=\left\{x_{1}, \ldots, x_{n}, y_{1}, \ldots, y_{s}\right\}$ with $x_{i}^{0} \leqq y_{j}^{0}$ and assume free boundary conditions in the time direction. Then we take as support of $\omega^{*}$, in a finite lattice, a set of strings $\gamma_{x i}^{-}$starting from $x_{i}$ and directed downward in the time direction, and a set of strings $\gamma_{y j}^{+}$starting from $y_{j}$ directed upward in the time direction. The corresponding disorder field is denoted by $D\left(\gamma_{x 1}^{-}, q_{1}, \ldots, \gamma_{x r}^{-}, q_{r}, \gamma_{y 1}^{+}\right.$, $\left.q_{r+1}, \ldots, \gamma_{y s}^{+}, q_{r+s}\right)$.

With this choice $\omega$ would have infinitely extended support in the thermodynamic limit and all expectation values involving the disorder field would vanish identically. Hence the correlation functions $S_{n, m}$ for such models are defined as thermodynamic limits of finite lattice correlations suitably normalized.

More precisely let $\mathcal{O}(\Gamma)$ denote either a Wilson loop or the scalar string variable defined in (2.13) or the fermion string variable

$$
\mathcal{O}_{B}\left(\Gamma_{x y}\right)=\bar{\psi}_{x} \prod_{b \in \Gamma_{x y}} \gamma_{b} e^{\frac{i}{N} A_{b}} \psi_{y} .
$$


Moreover let $\gamma_{x}$ denote a straight line in the time direction connecting the top to the bottom of the lattice and intersecting $x$, and denote by $D\left(\gamma_{x 1}, q_{1}, \ldots, \gamma_{x r}, q_{r}\right)$ the disorder field corresponding to a field $\omega$ given by

$$
\begin{aligned}
\omega_{\langle x y\rangle}^{*} & =q_{i} & & \langle x y\rangle \in \gamma_{i} \\
& =0 & & \text { otherwise }
\end{aligned}
$$

We then define, for $n=r+S$ :

$$
\begin{aligned}
S_{n, m}\left(\gamma_{x 1}^{-}, q_{1}, \ldots, \gamma_{x r}^{-}, q_{r}, \gamma_{y 1}^{+}, q_{r+1}, \ldots, \gamma_{y s}^{+}, q_{r+s}, \Gamma_{1} \ldots \Gamma_{m}\right) \\
=\lim _{\Lambda \uparrow \mathbf{Z}_{1 / 2}}\left[\left\langle D\left(\gamma_{x 1}^{-}, q_{1}, \ldots, \gamma_{y s}^{+}, q_{r+s}\right) \mathcal{O}\left(\Gamma_{1}\right) \ldots \mathcal{O}\left(\Gamma_{m}\right) G_{\partial \Lambda}\left(\sum_{i=1}^{r+s} q_{i}\right)\right\rangle_{\Lambda}\right. \\
\quad \times\left\langle D\left(\gamma_{x 1}, q_{1}, \ldots, \gamma_{x r}, q_{r}\right) G_{\partial \Lambda}\left(\sum_{i=1}^{r} q_{i}\right)\right\rangle_{\Lambda}^{-1 / 2} \\
\left.\quad \times\left\langle D\left(\gamma_{y 1}, q_{r+1}, \ldots, \gamma_{y s}, q_{r+s}\right) G_{\partial \Lambda}\left(\sum_{i=r+1}^{r+s} q_{i}\right)\right\rangle_{\Lambda}^{-1 / 2}\right]
\end{aligned}
$$

for $\sum_{i=1}^{n} q_{i}=0$, and the correlation functions of non-vanishing total charge are defined by sending a charge to infinity.

In (2.9) $G_{\partial \Lambda}(q)$ is a b.c. given by choosing a path $\gamma$ (as before) in $(\partial \Lambda)^{*}$ and setting

$$
A_{\langle x y\rangle}=q=\sum_{i}^{r} q_{i} \quad\langle x y\rangle \in \gamma^{*},
$$

setting $A_{\langle x y\rangle}=0$ in the links in the spatial boundary of $\partial \Lambda \notin \gamma^{*}$.

If cluster properties hold and correlation functions with non-vanishing total charge are zero, then soliton sectors can be constructed by means of Theorem 1.1 (see I). They are called stringlike soliton sectors and the reconstructed soliton field operators $s_{q}\left(\gamma_{x}\right)$ can be considered as a lattice version of the charged fields localized in cones studies in [7].

Our results for this model can be summarized in

Theorem 2.3. The model defined above for $\beta_{L}$ large, $\beta_{G} / \beta_{H}, \beta_{H} / \lambda, \beta_{B}$ small and

$$
\left.\beta_{G} \gg \min \left\{\left|\ln \left(\beta_{G} / \beta_{H}\right)\right|,\left|\ln \left(\beta_{H} / \lambda\right)\right|,\left|\ln \beta_{B}\right|\right)\right\} \equiv K_{B}
$$

possesses stringlike soliton sectors $\mathscr{H}_{q}$ labelled by the non-trivial elements $q$ of $\mathbf{Z}_{N}$.

Theorem 2.4. The soliton field operator $s_{1}\left(\gamma_{x}\right)$ couples the vacuum to a stable oneparticle state; in particular the two-point function

$$
\left\langle s_{1}\left(\gamma_{0}\right) \Omega, s_{1}\left(\gamma_{x}\right) \Omega\right\rangle=S_{2}\left(\gamma_{0}^{-},-1, \gamma_{x}^{+},+1\right)
$$

with $x=(t, \vec{x})$ has the following decay law:

$$
\left.\sum_{\vec{x}}\left\langle s_{1}\left(\gamma_{0}\right) \Omega, s_{1}\left(\gamma_{x}\right) \Omega\right)\right\rangle \underset{t \uparrow \infty}{\sim} e^{-m t}\left(1+e^{-\mu t}\right),
$$

where

$$
m=2 \pi^{2} \beta_{G}+\mathcal{O}\left(\frac{\beta_{\mathrm{G}}}{\beta_{H}^{1 / 2}}\right)+e^{-\mathcal{O}\left(K_{B}\right)}, \quad \mu=\mathcal{O}\left(K_{B}\right)
$$




\section{The Combined Low and High Temperature Expansion}

\subsection{The General Structure}

In this section we outline the general structure of a Combined Low and High Temperature (C.L.H.T.) expansion for mixed order-disorder correlation functions and prove the main convergence theorem. In Sect. 3.2 we show how such expansion applies to the concrete models discussed in Sect. 2.1.

Let $\theta$ denote the set of fields which represent orbits of the action of the hypergauge transformation on the basic fields and let $\sigma$ denote the $\mathscr{Z}$-valued field parametrizing the orbit, defined in Sect. 2.

As a first step we express the expectation value of disorder field and hypergauge invariant observables,

$$
\left\langle D(\omega) \prod_{e} \mathcal{O}\left(\omega ; \Gamma_{e}\right)\right\rangle
$$

in terms of the fields $\theta$ and $\sigma$.

By hypergauge invariance, $\sigma$ can appear only in the combination

$$
d \sigma+\omega \equiv v,
$$

and we obtain

$$
\begin{aligned}
\left\langle D(\omega) \prod_{e} \mathcal{O}\left(\omega ; \Gamma_{e}\right)\right\rangle_{\Lambda} & =\frac{\sum_{d v=d \omega} \int d \theta_{\Lambda} e^{-S_{\Lambda}(\theta, v)} \prod_{e} \mathcal{O}\left(v, \theta ; \Gamma_{e}\right)}{\sum_{v: d v=0} \int d \theta_{\Lambda} e^{-S_{\Lambda}(\theta, v)}} \\
& =\frac{\sum_{v: d v=d \omega} Z_{\Lambda}^{\Gamma}(v)}{\sum_{v: d v=0} Z_{\Lambda}(v)}
\end{aligned}
$$

where $d \theta_{A}$ denotes the measure induced on the orbit fields by the measure on the original basic fields of the theory and $\Gamma \equiv\left\{\Gamma_{e}\right\}$.

We now apply a low temperature expansion to treat the field $v$ and a high temperature expansion to treat the orbit fields both in the numerator and denominator of (3.2) (see Sect. 3.2 and the Appendix of I for more details).

Let us assume that as result the denominator of (3.2), i.e. the partition function $Z_{A}$, is expressed as the partition function of a polymer gas with polymers of two different types: $v$ - and $X$-polymers.

A $v$-polymer originates in the low-temperature expansion for the field $v$, and it has support on a set of cells whose dual is connected and closed.

An $X$-polymer originates in the high-temperature expansion for the orbit fields and it has support on a connected set of cells.

Then

$$
Z_{\Lambda}(v: d v=0)=\sum_{\left\{X_{1} \ldots X_{n}\right\}} Z\left(v_{1} \ldots v_{m}, X_{1}, \ldots, X_{n}\right),
$$

where $\left\{v_{1} \ldots v_{m}\right\}=v$ denote a set of $v$-polymers having the dual of their supports disjoint and $\left\{X_{1} \ldots X_{n}\right\}$ a set of $X$-polymers with disjoint supports. 
Besides ordinary $v$ - and $X$-polymers, the polymer representation of $Z_{\Lambda}^{\Gamma}(v: d v=d \omega)$ contains a $v^{\omega}$-polymer and a $X^{\Gamma}$-polymer such that every connected component of $\operatorname{supp} v^{\omega}$ (respectively $\operatorname{supp} X^{\Gamma}$ ) has nonvanishing intersection with $\operatorname{supp}(d \omega)$ (respectively $\Gamma$ ) and $d v^{\omega}=d \omega$.

We have

$$
Z_{\Lambda}^{\Gamma}(v: d v=d \omega)=\sum_{\left\{X_{1} \ldots X_{n}, X^{\Gamma}\right\}} Z\left(v_{1} \ldots v_{m}, v^{\omega}, X_{1} \ldots X_{n}, X^{\Gamma}\right),
$$

where $v=\left\{v_{1} \ldots v_{m}, v^{\omega}\right\}$, and the same disjointness condition on the supports as before holds.

We now combine together $v$-, $X-, v^{\omega}$-, $X^{\Gamma}$-polymers into "connected" clusters $C$ and $C^{\omega \Gamma}$ (see e.g. [12]).

A configuration $\left\{v_{1}, \ldots, v_{m}, X_{1}, \ldots, X_{n}\right\}$ is said to form a cluster on

$$
C=\operatorname{supp}\left\{\bigcup_{i=1}^{m} v_{i} \bigcup_{j=1}^{n} X_{j}\right\}
$$

iff supp $\left\{\bigcup_{i=1}^{m}\left(v_{i}\right)^{*} \bigcup_{j=1}^{n} X_{j}\right\}$ is connected.

A configuration $\left\{v_{1}, \ldots, v_{m}, v^{\omega}, X_{1}, \ldots, X_{n}, X^{\Gamma}\right\}$ is said to form a cluster on

$$
C^{\omega \Gamma}=\operatorname{supp}\left\{\bigcup_{i} v_{i} \bigcup_{j} X_{j} \cup v^{\omega} \cup X^{\Gamma}\right\}
$$

iff every connected component of

$$
\operatorname{supp}\left\{\bigcup_{i}\left(v_{i}\right)^{*} \bigcup_{j} X_{j} \bigcup\left(v^{\omega}\right)^{*} \bigcup X^{\Gamma}\right\}
$$

has non-vanishing intersection with $\operatorname{supp}\{(d \omega) \cup \Gamma\}$.

The activity of a cluster is defined by

$$
\begin{gathered}
Z(C)=\sum_{\left\{v_{1}, \ldots, v_{m}, X_{1} \ldots X_{n}\right\} \text { clusters on } C} Z\left(v_{1} \ldots v_{m}, X_{1} \ldots X_{n}\right), \\
Z\left(C^{\omega \Gamma}\right)=\sum_{\left\{v_{1}, \ldots, v_{m}, v^{\omega}, X_{1}, \ldots, X_{n}, X^{\Gamma}\right\} \text { clusters on } C^{\omega \Gamma}} Z\left(v_{1} \ldots v_{m}, v^{\omega}, X_{1} \ldots X_{n}, X^{\Gamma}\right) .
\end{gathered}
$$

Then one can rewrite

$$
(3.2)=\frac{\sum_{\left\{C_{r}, C^{\omega \Gamma}\right\}_{r}} \prod_{r} Z\left(C_{r}\right) Z\left(C^{\omega \Gamma}\right)}{\sum_{\left\{C_{r}\right\}} \prod_{r} Z\left(C_{r}\right)},
$$

where $\left\{C_{r}, C^{\omega \Gamma}\right\}$ denotes a set of clusters with disjoint supports.

Finally, using standard methods, the finite volume mixed correlation functions can be expressed in terms of the cluster expansion

$$
\begin{aligned}
& \left\langle D(\omega) \prod_{e} \mathcal{O}\left(\omega ; \Gamma_{e}\right)\right\rangle_{A} \\
& =\left.\frac{d}{d \alpha} \log \left[\left\{\sum_{\left\{C_{r}\right\}} \prod_{r} Z\left(C_{r}\right)\right\}+\alpha\left\{\sum_{\left\{C_{r}, C^{\omega \Gamma}\right\}} \prod_{r} Z\left(C_{r}\right) Z\left(C^{\omega \Gamma}\right)\right\}\right]\right|_{\alpha=0} \\
& =\sum_{\left\{\mathbf{C}, C^{\omega \Gamma}\right\}} \frac{\varphi_{T}\left(\mathbf{C}, C^{\omega \Gamma}\right)}{|\mathbf{C}| !} \prod_{C \in \mathbf{C}} Z(C) Z\left(C^{\omega \Gamma}\right),
\end{aligned}
$$


where $\left\{\mathbf{C}, C^{\omega \Gamma}\right\}$ denotes a collection of clusters in which a cluster $C$ can occur an arbitrary number of times and the cluster $C^{\omega \Gamma}$ occurs once; $\varphi_{T}\left(\mathbf{C}, C^{\omega \Gamma}\right)$ is a combinatorial factor which vanishes unless the set of clusters $\left\{\mathbf{C}, C^{\omega \Gamma}\right\}$ is connected and $|\mathbf{C}|$ ! is the number of identical permutations in $\{\mathbf{C}\}$.

More precisely the combinatorial factor $\varphi_{T}$ is defined as follows. Let $\sum_{\mathscr{G}}$ denote the sum over all connected graphs, $\mathscr{G}$, having as vertices the elements of $\left\{\mathbf{C}, C^{\omega \Gamma}\right\}$, let $\left\langle C, C^{\prime}\right\rangle$ denote a link in $\mathscr{G}$ connecting the clusters $C$ and $C^{\prime}$.

Define

$$
V\left(C, C^{\prime}\right)= \begin{cases}\infty & C \cap C^{\prime} \neq \phi \\ 0 & C \cap C^{\prime}=\phi,\end{cases}
$$

then

$$
\varphi_{T}\left(\mathbf{C}, C^{\omega \Gamma}\right)=\sum_{\mathscr{G}\left\langle C, C^{\prime}\right\rangle \in \mathscr{G}}\left(e^{-V\left(C, C^{\prime}\right)}-1\right) .
$$

To state the convergence theorem let us introduce some notations.

In the following $K$ will denote a generic constant and $K_{c}$ a large constant (both can have different values in different formulas) both independent on the coupling parameters.

$$
\begin{gathered}
\gamma \equiv \operatorname{supp} v, \quad X \cap v \equiv \operatorname{supp} X \cap \operatorname{supp} v, \\
v(X) \equiv v \quad \text { restricted to } X \cap v .
\end{gathered}
$$

If $F$ is a field of rank $k$ we set

$$
|F|_{p} \equiv \sum_{c_{k}}\left|F\left(c_{k}\right)\right|^{p}, \quad p \geqq 0 .
$$

Moreover given a field $F$ of rank $k$ and $f$ a function on sets of cells $Y$, we define

$$
\begin{gathered}
|F|_{p} \equiv \sum_{c_{k}}\left|F\left(c_{k}\right)\right|^{p}, \quad p \geqq 0, \\
\|f(Y)\|_{K}=\sup _{x \in A} \sum_{Y \text { is connected to } x}|f(Y)| e^{K|Y|} .
\end{gathered}
$$

The convergence theorem can then stated as follows:

Theorem 3.1. Suppose that there exist finite constants

$$
K_{v}, K_{1}, K_{2}, K_{e}
$$

with

$$
\begin{gathered}
K_{v} \gg K_{1} \gg 1, \quad K_{v} \gg\left|K_{2}\right|, \\
\left|Z\left(v=\left\{v_{1} \ldots v_{m}, v_{\omega}\right\} ; X=\left\{X_{1} \ldots X_{n}, X^{\Gamma}\right\}\right)\right| \leqq \prod_{e} K_{e} e^{-K_{v}|v|_{p}} e^{-K_{1}|X \backslash X \cap v|} e^{K_{2}|v(X)|_{p}},
\end{gathered}
$$

with $p \geqq 0$, and $p \geqq 1$ if $\mathscr{Z}$ is non-compact.

Then:

1) the expansion (3.6) converges with a bound

$$
\left|\left\langle D(\omega) \prod_{e} \mathcal{O}\left(\omega ; \Gamma_{e}\right)\right\rangle\right| \leqq K \frac{1}{1-\|Z(C)\|_{k}}\left\|Z\left(C^{\omega \Gamma}\right)\right\|_{k}
$$


for some $k>1$,

2) the thermodynamic limit exists,

3) clustering holds for all correlation functions,

4) all the limits (1.3) vanish.

From Theorem 1.1 it follows that the models to which the C.L.H.T. expansion applies posses soliton sectors labelled by the non-trivial elements of $\mathscr{Z}$, in the parameter region where the bound (3.7) hold.

The cluster expansion outlined above is somehow similar to the "mean field expansion" in the lattice approximation [8]. The main difference is that we use a true high temperature expansion for the orbit fields instead of an expansion around the gaussian as in [8]. Although the C.L.H.T. expansion does not easily generalize to the continuum, it has the advantage, for lattice field theories, of nicer decoupling properties between the clusters which strongly simplify the particle analysis (see Sect. 4).

A similar expansion for the non-compact abelian Higgs model has been discussed in [9] and for gauge theories with discrete finite center has been sketched in $[10]$.

Proof of Theorem 3.1. We first prove that, if the bound (3.7) holds with $K_{v}, K_{1 X}$ sufficiently large, then there exist $K_{c} \ll K_{1}$ such that

$$
\begin{gathered}
|Z(C)| \leqq \exp -\left(K_{1}-K_{c}\right)|C| \\
\left|Z\left(C^{\omega}\right)\right| \leqq K \prod_{e} K_{e} e^{-\left(K_{1}-K_{c}\right)\left|C^{\omega \Gamma}\right|}\left|Z\left(v_{\min }^{\omega}\right)\right| \cdot e^{\left(K_{1}+K_{2}+K_{c}\right)\left|\gamma_{\min }^{\omega}\right|},
\end{gathered}
$$

where $v_{\min }^{\omega}$ is a configuration of $v^{\omega}$ with the highest value of $\left|Z\left(v^{\omega}\right)\right| e^{\left(K_{1}+K_{2}+K_{c}\right)\left|\gamma^{\omega}\right|}$ in the family of the clusters on $C^{\omega \Gamma}$, and $\gamma_{\min }^{\omega} \equiv \operatorname{supp} v_{\min }^{\omega}$.

We first sum in (3.4) over $\mathscr{Z} \backslash\{0\}$ in all the cells in the support of the configurations of $v$ in $C$.

For $K_{v}$ sufficiently large

$$
\sum_{z \in \mathscr{Z} \backslash\{0\}} \exp -\left(K_{v}-K_{2}\right)|z|^{p} \leqq K e^{-\left(K_{v}-K_{2}\right)},
$$

so that using $|\gamma| \geqq|X \cap v|$ one obtains

$$
|Z(C)| \leqq K\left[\sum_{\substack{\left\{\gamma_{1} \ldots \gamma_{m}, X_{1} \ldots X_{n}\right\}: \\\left\{v_{1} \ldots v_{m}, X_{1} \ldots X_{n}\right\} \text { clusters on } C}} \prod_{i=1}^{m} e^{-\left(K_{v}-K_{1}-K_{2}\right)\left|\gamma_{i}\right|} \cdot \prod_{j=1}^{n} e^{-K_{1}\left|X_{j}\right|}\right] .
$$

We now observe that $\bigcup_{j}\left|\gamma_{i}\right| \bigcup_{j}\left|X_{j}\right| \geqq|C|$ since $X_{j}$ can overlap $\gamma_{i}$.

Hence we can extract an overall exponential factor proportional to $|C|$. We then substitute

$$
\left\{\gamma_{i}, X_{j}\right\}:\left\{v_{i}, X_{j}\right\} \text { clusters on } C
$$

with

$$
\{\gamma, \mathbf{X}\} \text { : each } X \text {, each } \gamma \text { is connected to } C
$$


where $\{\gamma, \mathbf{X}\}$ denotes families of $\gamma, X$ in which $\gamma, X$ can occur an arbitrary number of times, and divide by the number of identical permutations, $|\gamma|$ ! $|\mathbf{X}|$ !.

Hence for some $K_{c} \ll K_{1}$ still large,

$$
\begin{aligned}
|Z(C)| \leqq & e^{-\left(K_{1}-K_{c}\right)|C|} \\
& \times\left[\sum_{\substack{\{\gamma, \mathbf{X}\}: \\
\text { each } \gamma, \text { each } X \text { is connected in } C}} \frac{1}{|\gamma| !} \frac{1}{|\mathbf{X}| !} \prod_{X} e^{-K_{c}|X|} \prod_{\gamma} e^{-K_{c}|\gamma|}\right] .
\end{aligned}
$$

It is a standard result [8] that, for $K_{c}$ sufficiently large

$$
\sum_{\substack{\gamma: \\ \text { each } \gamma \text { is connected to } c}} \frac{1}{|\gamma| !} \prod_{\gamma \in \gamma} e^{K_{c}|\gamma|} \leqq e^{-\| e^{-K_{c}|\gamma| \|_{0}|C|},}
$$

and a similar bound holds for the $X$-polymers.

From these bounds and Eq. (3.11), Eq. (3.9i) follows. Analogously

$$
\left|Z\left(C^{\omega \Gamma}\right)\right| \leqq \prod_{e} K_{e} \cdot \sum_{v^{\omega} \subset C^{\omega \Gamma}}\left|Z\left(v^{\omega}\right)\right| e^{\left(K_{1}+K_{2}+K_{c}\right)\left|\gamma^{\omega \mid}\right|} \cdot e^{-\left(K_{1}-K_{c}\right)\left|C^{\omega \Gamma}\right|} .
$$

We observe that

$$
\left\{v^{\omega}:\left|\gamma^{\omega}\right| \leqq \gamma_{\min }^{\omega} \mid\right\} \leqq e^{K\left|\gamma_{\min }^{\omega}\right|},
$$

where $K$ may now depend on the charges of $d \omega$, and for $\left|\gamma^{\omega}\right|>\left|\gamma_{\min }^{\omega}\right|$,

$$
\left|\frac{Z\left(v^{\omega}\right) e^{\left(K_{1}+K_{2}+K_{c}\right)\left|\gamma^{\omega \mid}\right|}}{Z\left(v_{\min }^{\omega}\right) e^{\left(K_{1}+K_{2}+K_{c}\right)\left|\gamma_{\text {min }}\right|}}\right| \leqq e^{-\mathscr{O}\left(K_{v}\right)\left[\left|\gamma^{\omega}\right|-\left|\gamma_{\min }^{\omega}\right|\right]} .
$$

Therefore,

$$
\sum_{v^{\omega} C C^{\omega \omega}}\left|Z\left(v^{\omega}\right)\right| e^{\left(K_{1}-K_{2}+K_{c}\right) \mid \gamma^{\omega \omega}} \leqq\left|Z\left(v_{\min }^{\omega}\right)\right| e^{\left(K_{1}+K_{2}+K_{c}\right)\left|\gamma_{\min }^{\omega}\right|},
$$

and we obtain (3.9ii).

Using Eq. (3.9) the convergence of the expansion (3.6) and the estimate (3.8) are straightforward modifications of the Appendix in [8] and are omitted.

Remark 3.2. It follows from the proof, that one can easily weaken some of the assumptions of Theorem 3.1.

We can admit several type of $v$ - and $X$-polymers and we can also consider on the support of polymers more general constrains than connectedness constraints in Eq. (3.3), provided the following condition is satisfied.

Let us denote by "connectedness" this weaker constraint and by $P$ a generic polymer, occurring in the expansion, then for any polymer $P^{\prime}$, for $K_{c}$ sufficiently large, there must exist a constant $K$ such that

$$
\sum_{P: P \text { "connected" to } P^{\prime}} e^{-K_{c}|P|} \leqq K\left|P^{\prime}\right| .
$$

As an example in $d=3$ let the support of the polymer be given by closed curves, then we can consider "connected"= "linked".

\subsection{The Models (proof of Theorem 2.1)}

In this section we identify the $v$ - and $X$-polymers in the models described in Sect. 2.1 and prove the bound (3.7) on their activity $\left(v^{\omega}-, X^{\Gamma}\right.$-polymers can be 
treated in a similar way), hence establishing the existence of soliton sectors, thanks to Theorems 1.1, 3.1.

As one can see from the explicit discussion, such a procedure can be easily generalized to a large variety of models (satisfying condition A of I).

a) $\phi_{2}^{4}$

The proof of Theorem 1a is sketched in Appendix 1 of I and the missing details are easily filled following the method used here for the model $b$ ).

In particular we obtain

$$
K_{1}=\mathcal{O}\left(\left|\ln \beta_{H} / \lambda\right|\right), \quad K_{2}=-\mathcal{O}\left(\left|\ln \beta_{H} / \lambda^{1 / 2}\right|\right), \quad K_{v}=2 \beta_{H} .
$$

b) Non-compact $(U(1) \text { Higgs })_{3}$.

To rewrite the partition function of the model in terms of defects some care is needed for the gauge fixing term. Proceeding as in [8] we finally obtain

$$
\begin{aligned}
Z_{\Lambda}= & \sum_{v: d v=0} \int \prod_{x \in \Lambda} d r_{x} \prod_{\langle x y\rangle \in \Lambda} d \theta_{\langle x y\rangle} \exp \left\{-\left[\beta_{G} \sum_{p \in \Lambda}\left(d \theta_{p}+2 \pi v_{p}\right)^{2}\right.\right. \\
& \left.\left.+\frac{\beta_{H}}{2} \sum_{\langle x y\rangle \in \Lambda}\left(r_{x}-e^{i \theta\langle x y\rangle} r_{y}\right)^{2}+\lambda \sum_{x \in \Lambda}\left(r_{x}^{2}-1\right)^{2}\right]\right\} \prod_{\langle x y\rangle \in \partial \Lambda} \delta\left(\theta_{x y}\right),
\end{aligned}
$$

where $r_{x}=\left|\phi_{x}\right| \in \mathbf{R}_{+}, \theta_{\langle x y\rangle} \in U(1) \cong(-\pi, \pi)$ and the unessential term $\log r_{x}$ was omitted.

Define the unnormalized measures

$$
d \tilde{v}\left(r_{x}\right)=d r_{x} e^{-\lambda\left(r_{x}^{2}-1\right)^{2}}, \quad d \tilde{v}\left(\theta_{\langle x y\rangle}\right)=d \theta_{\langle x y\rangle} e^{-\beta_{H}\left(1-\cos \theta_{\langle x y\rangle}\right)} .
$$

The corresponding normalized measures are denoted by $d v\left(r_{x}\right), d v\left(\theta_{\langle x y\rangle}\right)$ and are strongly peaked around $r=1, \theta=0$ for $\lambda, \beta_{H}$ large.

Define the functions

$$
\begin{gathered}
R_{\langle x y\rangle}=\exp \left\{-\frac{\beta_{H}}{2}\left[\left(r_{x}-e^{i \theta_{\langle x y\rangle}} r_{y}\right)^{2}-\left(1-e^{i \theta_{\langle x y\rangle}}\right)^{2}\right]\right\}-1, \\
R_{p}=\exp \left\{-\frac{\beta_{G}}{2}\left[\left(d \theta_{p}+2 \pi v_{p}\right)^{2}-\left(2 \pi v_{p}\right)^{2}\right]\right\}-1 .
\end{gathered}
$$

These functions are small with high probability, with respect to the measures $d v\left(r_{x}\right)$, $d v\left(\theta_{\langle x y\rangle}\right)$, in large regions and can be treated with high temperature techniques. Defining

$$
Z_{0}(\Lambda)=\prod_{x \in \Lambda} \int d \tilde{v}\left(\varrho_{x}\right) \prod_{\langle x y\rangle \in \Lambda} \int d \tilde{v}\left(\theta_{\langle x y\rangle}\right)
$$

one can rewrite

$$
\begin{gathered}
Z_{\Lambda}=Z_{0}(\Lambda) \sum_{v: d v=0} \prod_{p \in \Lambda} e^{-\frac{\beta_{G}}{2}\left(2 \pi v_{p}\right)^{2}} \int \prod_{x \in \Lambda} d v\left(r_{x}\right) \\
\cdot \prod_{\langle x y\rangle \in \Lambda} d v\left(\theta_{\langle x y\rangle}\right) \prod_{p \in \Lambda}\left(R_{p}+1\right) \prod_{\langle x y\rangle \in \Lambda}\left(R_{\langle x y\rangle}+1\right) \prod_{\langle x y\rangle \in \partial \Lambda} \delta\left(\theta_{\langle x y\rangle}\right)
\end{gathered}
$$

An $X$-polymer has support on a set of links, $X^{1}$, and plaquettes, $X^{2}$, such that $X^{1} \cup X^{2}$ is connected and we define its activity by

$$
\begin{gathered}
Z(X)=\int \prod_{x \in X} d v\left(r_{x}\right) \prod_{\langle x y\rangle \in X} d v\left(\theta_{\langle x y\rangle}\right) \prod_{\langle x y\rangle \in X \cap \partial A} \delta\left(\theta_{\langle x y\rangle}\right) \\
\cdot \prod_{\langle x y\rangle \in X^{1}} R_{\langle x y\rangle} \prod_{p \in X^{2}} R_{p} .
\end{gathered}
$$


A v-polymer has support on a set of plaquettes whose dual is closed and we define its activity by

$$
Z(v)=e^{-2 \pi^{2} \beta_{G}|v|_{2}} .
$$

With such definitions one can easily show that the representation (3.4) holds with

$$
Z\left(X_{1} \ldots X_{n}, v_{1} \ldots v_{m}\right)=\prod_{i=1}^{n} Z\left(X_{i}\right) \prod_{j=1}^{m} Z\left(v_{j}\right) .
$$

The bound (3.7) can be obtained as follows.

We substitute $d v\left(r_{x}\right)$ in $Z\left(X_{i}\right)$ with a gaussian measure with mean 1 and covariance $1 / 4 \lambda$, using the inequality

$$
\left(r_{x}^{2}-1\right)^{2} \geqq 2\left(r_{x}-1\right)^{2} .
$$

Similarly we substitute $d v\left(\theta_{\langle x y\rangle}\right)$ with a gaussian measure with mean 0 and covariance $4 / \beta_{H}$ using the inequality

$$
\beta_{H}\left(1-\cos \theta_{\langle x y\rangle}\right) \geqq \frac{1}{8} \beta_{H} \theta_{\langle x y\rangle}^{2}, \quad \theta_{\langle x y\rangle} \in(-\pi, \pi) .
$$

We denote by $d \tilde{\mu}_{G}\left(r_{x}\right)\left(d \mu_{G}\left(r_{x}\right)\right)$ the unnormalized (normalized) gaussian measure for $r_{x}$.

The ratio $\int d \tilde{v}\left(r_{x}\right) / \int d \tilde{\mu}_{G}\left(r_{x}\right)$ is evaluated by means of a Jensen inequality:

for $\lambda$ large.

$$
\begin{aligned}
\frac{\int d \tilde{v}\left(r_{x}\right)}{\int d \tilde{\mu}_{G}\left(r_{x}\right)} & =\int d \mu_{G}\left(r_{x}\right)\left(1-\chi\left(-r_{x}\right)\right) e^{-\lambda\left[\left(r_{x}^{2}-1\right)^{2}-2\left(r_{x}-1\right)^{2}\right]} \\
& \leqq e^{-\lambda\left\langle\left(r_{x}^{2}-1\right)^{2}-2\left(r_{x}-1\right)^{2}\right\rangle_{G}}-e^{-\mathcal{O}(\lambda)}=\mathcal{O}(1)
\end{aligned}
$$

Similar estimates hold for $\theta$.

Then by Hölder's inequalities and gaussian estimates one easily obtains:

$$
|\mathrm{Z}(\mathrm{X})| \leqq \mathcal{O}\left(\frac{\beta_{H}}{\lambda}\right)^{\left|X^{1}\right|} \mathcal{O}\left(\frac{\beta_{G}}{\beta_{H}}\right)^{\left|X^{2} \backslash X^{2} \cap v\right|} e^{\mathcal{O}\left(\frac{\beta_{G}^{2}}{\beta_{H}}\right)|v|_{2}}
$$

Hence one can identify:

$$
\begin{gathered}
K_{1}=\min \left[\mathcal{O}\left(\left|\ln \beta_{H} / \lambda\right|\right), \mathcal{O}\left(\left|\ln \beta_{G} / \beta_{H}\right|\right)\right], \\
K_{2}=\mathcal{O}\left(\beta_{G}^{2} / \beta_{H}\right), \quad K_{v}=2 \pi^{2} \beta_{G} .
\end{gathered}
$$

Remark 3.3. If $v(X)$ has charge \pm 1 on $X^{2} \cap v$, one obtains a stronger bound where $K_{2}$ can be substituted by

$$
\tilde{K}_{2}=-\mathcal{O}\left(\left|\ln \beta_{G} / \beta_{H}^{1 / 2}\right|\right)
$$

c) $(S U(N) \text { Higgs })_{3}$.

For simplicity we discuss the limiting $\lambda=\infty$ model. To analyze the model we first go in the unitary gauge, i.e. we make the substitution

$$
\left(\phi_{x}, U_{H}\left(g_{\langle x y\rangle}\right) \phi_{y}\right) \rightarrow\left(\phi_{0}, U_{H}\left(g_{\langle x y\rangle}\right) \phi_{0}\right)
$$

for some fixed $\phi_{0} \in V_{H}$.

Then one can write the partition function in terms of defects as:

$$
\begin{gathered}
Z_{\Lambda}=\sum_{v: d v=0} \int_{\langle x y\rangle \in \Lambda} \prod_{\langle x y\rangle} d h_{\langle x y\rangle \in \Lambda} e^{\beta_{H}\left[\operatorname{Re}\left(\phi_{0}, U_{H}\left(h_{\langle x p}\right) \phi_{0}\right)-1\right]} \\
\prod_{p \in \Lambda} e^{\beta_{G}\left[\operatorname{Re} \chi\left(h_{\partial p}\right) \chi\left(v_{p}\right)-1\right]}
\end{gathered}
$$


where $h_{\langle x y\rangle}$ is a gauge field taking values in $S U(N) / \mathbf{Z}_{N} \subset S U(N)$ and $d h_{\langle x y\rangle}$ is the Haar measure on $S U(N) / \mathbf{Z}_{N}$.

We define

$$
d \tilde{v}\left(h_{\langle x y\rangle}\right)=d h_{\langle x y\rangle} e^{\beta_{H}\left[\operatorname{Re}\left(\phi_{0}, U_{H}\left(h_{\langle x y\rangle}\right) \phi_{0}\right)-1\right]}
$$

and

$$
R_{p}=e^{\beta_{G}\left[\operatorname{Re} \chi\left(h_{\partial p}\right) \chi\left(v_{p}\right)-\operatorname{Re} \chi\left(v_{p}\right)\right]}-1 .
$$

The polymer $X$ is a connected set of plaquettes and its activity is defined by

$$
Z(X)=\int \prod_{\langle x y\rangle \in X} d v\left(h_{\langle x y\rangle}\right) \prod_{p \in X} R_{p} .
$$

By Hölder's inequality

$$
|Z(X)| \leqq \prod_{p \in X}\left[\int_{\langle x y\rangle \in \hat{o} p} d v\left(h_{\langle x y\rangle}\right)\left|R_{p}\right|^{2 r}\right]^{1 / 2 r}
$$

where

$$
r=\#\{\text { plaquettes sharing a link }\} .
$$

We now split the integration of $h_{\langle x y\rangle}$ in a neighbourhood $\mathscr{N}$ of the identity of $S U(N) / \mathbf{Z}_{N}$ and its complement $\mathscr{N}^{c}$. Let $q=N^{2}-1$ and let $\left\{r^{a}\right\}_{a=1}^{q}$ denote the generators of the Lie algebra of $S U(N)$.

If $\mathscr{N}$ is sufficiently small, the exponential map

$$
\begin{gathered}
\exp : R^{d} \rightarrow S U(N) \\
A_{\langle x y\rangle}^{a} \rightarrow e_{\langle x y\rangle}^{A} \equiv \exp \left(\sum_{a} A_{\langle x y\rangle}^{a} \tau^{a}\right) \equiv h_{\langle x y\rangle}
\end{gathered}
$$

exists. For our purposes we define

$$
\mathscr{N}=\left\{h_{\langle x y\rangle}:\left(A_{\langle x y\rangle}^{a}\right)^{2} \leqq \frac{1}{\beta_{G}}\right\}
$$

which is well defined for $\beta_{G}$ large enough.

The following inequalities occur for $\beta_{G}$ sufficiently large:

$$
\begin{gathered}
{\left[1-\operatorname{Re}\left(\phi_{0}, U_{H}\left(e^{A}\right) \phi_{0}\right)\right] \mid \mathcal{N} \leqq \frac{1}{2}\left(\phi_{0}, A_{H}^{2} \phi_{0}\right),} \\
{\left[1-\operatorname{Re}\left(\phi_{0}, U_{H}(h) \phi_{0}\right)\right] \mid \mathscr{N}^{c} \geqq \mathcal{O}\left(\beta_{G}^{-1}\right),}
\end{gathered}
$$

where

$$
A_{H}=\sum_{a=1} \tau_{H}^{a} A^{a}
$$

and $\left\{\tau_{H}^{a}\right\}$ are the generators of the Lie algebra of the representation $U_{H}$ of $S U(N) / \mathbf{Z}_{N}$ on $V_{H}$.

The terms in $\mathscr{N}$ are evaluated by:

1. substituting the measure

$$
d \tilde{v}(A)\left|\exp ^{-1} \mathscr{N}=d^{q} A \exp \beta_{H}\left[\operatorname{Re}\left(\phi_{0}, U_{H}\left(e^{A}\right) \phi_{0}\right)-1\right]\right| \exp ^{-1} \mathscr{N}
$$


with the (unnormalized) gaussian measure $d \tilde{\mu}_{G}(A)$ with mean zero and covariance $\left[\beta_{H}\left(\phi_{0}, \tau_{H}^{a} \tau_{H}^{b} \phi_{0}\right)\right]^{-1}$, using the fact that

$$
\exp ^{*}[d v(h) \mid \mathcal{N}]=\prod_{1}^{q}\left(1+\mathcal{O}\left(\operatorname{Tr} A^{2}\right)\right) d \tilde{v}(A) \mid \exp ^{-1} \mathcal{N},
$$

2. expanding the exponential in $R_{p}$ in terms of $\cos A, \sin A$, then

3. expanding $\cos A$ and $\sin A$ in terms of $A$ and performing gaussian estimates. The terms in $\mathscr{N}^{c}$ are estimated using (3.20ii).

The main bounds are

$$
\begin{aligned}
\frac{\int_{\exp ^{-1} \mathcal{\mathcal { V }}} d \tilde{v}(A)}{\int d \tilde{\mu}_{G}(A)}= & \int d \mu_{G}(A)\left(1+\mathcal{O}\left(\operatorname{Tr} A^{2}\right)\right) e^{-\beta_{H}\left(\phi_{0},\left(1-\cos A_{H}-\frac{1}{2} A_{H}^{2}\right) \phi_{0}\right)} \\
& \cdot\left\{1+\left[\prod_{a} \chi\left(1-\left(A^{a}\right)^{2} \beta_{G}\right)-1\right]\right\} \sim \mathcal{O}(1)+\mathcal{O}\left(\frac{1}{\beta_{H}}\right)-e^{-\mathcal{O}\left(\frac{\beta_{H}}{\beta_{G}}\right),} \\
& \int_{\langle x y\rangle \in \partial p} d \mu_{G}(A)\left(1+\mathcal{O}\left(\operatorname{Tr} A^{2}\right)\right)\left|R_{p}\left(e^{A}, v\right)\right|^{2} \\
& \leqq\left\{\begin{array}{ll}
\mathcal{O}\left(\frac{\beta_{G}}{\beta_{H}}\right)^{2 r}, & v_{p}=0 \\
\mathcal{O}\left(\frac{\beta_{G}}{\beta_{H}^{1 / 2}}\right)^{2 r}, & v_{p} \neq 0
\end{array},\right. \\
& \int_{\mathbb{N} c} d \tilde{v}(h)\left|R_{p}\right|^{2 r} \leqq e^{\mathcal{O}\left(\frac{\beta_{H}}{\beta_{G}}\right)} \operatorname{Max} R_{p} \mid \mathscr{N}^{c} \leqq \begin{cases}\mathcal{O}(1) e^{-\mathcal{O}\left(\frac{\beta_{H}}{\beta_{G}}\right),} & v_{p}=0 \\
e^{\mathcal{O}\left(\beta_{G}\right)} e^{-\mathcal{O}\left(\frac{\beta_{H}}{\beta_{G}}\right)} & v_{p} \neq 0\end{cases}
\end{aligned}
$$

Hence for $\beta_{G} / \beta_{H}^{1 / 2} \ll 1$ all contributions coming from $\mathscr{N}^{c}$ are negligible. Therefore

$$
\begin{gathered}
Z(v)=\exp -\beta_{G}|1-\chi(v)|_{1}, \\
|Z(X)| \leqq \mathcal{O}\left(\frac{\beta_{G}}{\beta_{H}}\right)^{|X| X \cap v \mid} \mathcal{O}\left(\frac{\beta_{G}}{\beta_{H}^{1 / 2}}\right)^{|v(X)|_{0}} .
\end{gathered}
$$

Hence we can identity

$$
Z\left(X_{1} \ldots X_{n}, v_{1} \ldots v_{m}\right)=\prod_{i=1}^{n} \prod_{j=1}^{m} Z\left(X_{i}\right) \prod Z\left(v_{j}\right)
$$

and

$$
K_{1}=\mathcal{O}\left(\left|\ln \beta_{G} / \beta_{H}\right|\right), \quad K_{2}=-\mathcal{O}\left(\left|\ln \beta_{G} / \beta_{H}^{1 / 2}\right|\right), \quad K_{v}=\mathcal{O}\left(\beta_{G}\right) .
$$

\section{Particle Structure Analysis for Local Solitons}

Proof of Theorem 2.2. In this section we consider the two point function of the soliton field operator $s_{1}(x)$, i.e. $S_{2}(x,-1, y, 1)$. We study its long distance behaviour by means of the excitation expansion proposed in [3] to analyze the particle structure of lattice field theories. 
Equation (3.6) expresses the two point function in terms of the convergent cluster expansion:

$$
\left\langle s_{1}(x) \Omega, s_{1}(y) \Omega\right\rangle=\sum_{\left\{\mathbf{C}, C_{x y}\right\}} \frac{\varphi_{T}\left(\mathbf{C}, C_{x y}\right)}{|\mathbf{C}| !} Z\left(C_{x y}\right) \prod_{C \in \mathbf{C}} Z(C),
$$

where we set $C^{\omega} \equiv C_{x y}$.

A cell in $\left\{\mathbf{C}, C_{x y}\right\}$ is called regular if:

1. it is orthogonal to the time axis;

2. it occurs only in $C_{x y}$;

3. there are no other cells having the same projection on the time axis;

4. it is not connected to other cells.

The connected complements of the regular cells in $\left\{\mathbf{C}, C_{x y}\right\}$ are called excitations and denoted by $\varepsilon$. Denote by $\pi$ the projection on the time axis, by $l(\varepsilon)$ the cardinality of $\varepsilon$, and set

$$
|\varepsilon| \equiv l(\varepsilon)-\pi(\varepsilon)
$$

Two excitations $\varepsilon_{1}, \varepsilon_{2}$ are called compatible if

$$
\pi\left(\varepsilon_{1}\right) \cap \pi\left(\varepsilon_{2}\right)=\varnothing
$$

and an excitation $\varepsilon$ is said to be allowed in $\left[x^{0}, y^{0}\right]$ if there exists a configuration $\left\{\mathbf{C}, C_{x y}\right\}$, with $x, y$ having time coordinates $x^{0}, y^{0}$, such that $\varepsilon$ is an excitation of such a configuration.

There is a one-to-one correspondence between the configurations $\left\{\mathbf{C}, C_{x y}\right\}$, occurring in the cluster expansion for $\sum_{\vec{x}}\left\langle s_{1}(0) \Omega, s_{1}(x) \Omega\right\rangle$ with $x=(t, \vec{x})$, and the sets $\left\{\varepsilon_{1} \ldots \varepsilon_{n}\right\}$ of excitations compatible, allowed in $[0, t]$ (see [3]).

Since the configuration with no excitations is the dual of a straight line, all the cells which contribute to it are disjoint. Denote by $Z_{\omega}$ the contribution of one such cell and define $C(\varepsilon)=C \cap \varepsilon, C_{0 x}(\varepsilon)=C_{0 x} \cap \varepsilon$. One easily realizes that, for $\left\{\varepsilon_{1} \ldots \varepsilon_{n}\right\}$ as above, the following factorization occurs:

$$
\varphi_{T}\left(\mathbf{C}, C_{0 x}\right)=\prod_{i=1}^{n} \varphi_{T}\left(\mathbf{C}\left(\varepsilon_{i}\right), C_{0 x}\left(\varepsilon_{i}\right)\right)
$$

Defining the activity of an excitation by:

$$
\zeta(\varepsilon)=\prod_{C(\varepsilon) \in \mathbf{C}(\varepsilon)} Z(C(\varepsilon)) Z\left(C_{0 x}(\varepsilon)\right)\left(\frac{\varphi_{T}\left(\mathbf{C}(\varepsilon), C_{0 x}(\varepsilon)\right)}{|\mathbf{C}(\varepsilon)| !}\right) Z_{\omega}^{-|\pi(\varepsilon)|},
$$

one has the representation

$$
\sum_{\vec{x}}\left\langle s_{1}(0) \Omega, s_{1}(x) \Omega\right\rangle=Z_{\omega}^{t} \sum_{\left\{\varepsilon_{1} \ldots \varepsilon_{n}\right\}} \prod_{i=1}^{n} \zeta\left(\varepsilon_{i}\right)=e^{t P[0, t]},
$$

where $P(0, t)$ is the pressure of the gas of excitations constrained in the interval $[0, t]$, obtained by formal exponentation and given by

$$
t P_{[0, t]}=t \ln Z_{\omega}+\sum_{\varepsilon} \prod_{\varepsilon \in \varepsilon} \zeta(\varepsilon) \frac{\varphi_{T}(\varepsilon)}{|\varepsilon| !} .
$$


Theorem 4.1. Assume that for $v$ taking value 1 on $X \cap v$ bounds (3.7) hold even if $K_{2}$ is replaced by a $\tilde{K}_{2}<0$.

Then, under the assumptions of Theorem 3.1 and for $\left|\widetilde{K}_{2}\right|$ large enough, the activity of the excitation is bounded by

$$
|\zeta(\varepsilon)| \leqq e^{-\left(K_{1}-\left|K_{2}\right|-K_{c}\right)|\varepsilon|}
$$

and the expansion (4.6) converges.

Proof. From estimate (4.7) convergence of the expansion (4.6) is a standard fact for $K_{1}$ large enough. To obtain (4.7) we bound separately each term in (4.4). Let $c$ a cell contributing to a configuration with no excitations and set $Z_{\omega}=Z_{\omega}(c)$. Then $Z_{\omega}(c)$ has two contributions: one corresponds to the configurations in which $c$ is only in the support of $v^{\omega}$ and one to the configurations in which $c$ is also the support of a $X$-polymer, i.e.

$$
Z_{\omega}(c)=Z\left(v^{\omega}(c)\right)+Z\left(v^{\omega}(c), X=c\right) .
$$

From the assumptions of the theorem it follows:

$$
Z_{\omega}(c)=Z\left(v^{\omega}(c)\right)\left(1+0\left(e^{\tilde{K}_{2}}\right)\right),
$$

and for $\left|\tilde{K}_{2}\right|$ large enough there exists a constant $K$ such that

$$
Z_{\omega}^{-1}(c) \leqq K Z\left(v^{\omega}(c)\right)^{-1}
$$

By a standard polymer estimate [3]

$$
\left|\frac{\varphi_{T}\left(\mathbf{C}(\varepsilon), C_{0 x}(\varepsilon)\right)}{\mid \mathbf{C}(\varepsilon) !}\right| \leqq e^{K|\varepsilon|},
$$

and from Eq. (3.9),

$$
\begin{gathered}
|Z(C(\varepsilon))| \leqq e^{-\left(K_{1}-K_{c}\right)|C(\varepsilon)|} \\
\left|Z\left(C_{0 x}(\varepsilon)\right)\right| \leqq\left|Z\left(v_{\min }^{\omega}(\varepsilon)\right)\right| e^{\left(K_{1}+K_{2}+K_{c}\right)\left|\gamma_{\min }^{\omega}(\varepsilon)\right|} e^{-\left(K_{1}-K_{c}\right)\left|C_{0 x}(\varepsilon)\right|} .
\end{gathered}
$$

Since $v=1$ at least on the cells corresponding to $\pi(\varepsilon)$,

$$
\left|Z\left(v_{\min }^{\omega}(\varepsilon)\right)\right| Z_{\omega}^{-|\pi(\varepsilon)|} \leqq e^{-\mathcal{O}\left(K_{v}\right)\left[\left|\gamma_{\min }(\varepsilon)\right|-|\pi(\varepsilon)|\right]} K^{|\pi(\varepsilon)|},
$$

so that

$$
\left|Z\left(v_{\min }^{\omega}(\varepsilon)\right)\right| e^{\left(K_{1}+K_{2}+K_{c}\right)\left|\gamma_{\min }^{\omega}(\varepsilon)\right|} Z_{\omega}^{-|\pi(\varepsilon)|} \leqq e^{\left(K_{1}+K_{2}+K_{c}\right)|\pi(\varepsilon)|} .
$$

From the definition of $l(\varepsilon)$ we also have

$$
\prod_{C(\varepsilon) \in \mathbf{C}(\varepsilon)} e^{-\left(K_{1}-K_{c}\right)|C(\varepsilon)|} e^{-\left(K_{1}-K_{c}\right)\left|C_{0 x}(\varepsilon)\right|}=e^{-\left(K_{1}-K_{c}\right)[|\varepsilon|+|\pi(\varepsilon)|]} .
$$

Using $|\pi(\varepsilon)| \leqq|\varepsilon|$, Eqs. (4.4) and (4.8-10) yield

$$
\begin{aligned}
|\zeta(\varepsilon)| & \leqq e^{-\left(K_{1}-K_{c}\right)[|\pi(\varepsilon)|+|\varepsilon|]} e^{\left(K_{1}+K_{2}+K_{c}\right)|\pi(\varepsilon)|} \\
& =e^{\left(K_{2}+K_{c}\right)|\pi(\varepsilon)|} e^{-\left(K_{1}-K_{c}\right)|\varepsilon|} \\
& \leqq e^{-\left(K_{1}-K_{c}-\left|K_{2}\right|\right)|\varepsilon|}
\end{aligned}
$$

From Eqs. (4.6), (4.7) the proof of Theorem 2.2 easily follows using the techniques of [3] and the estimates of Sect. 3.2. 


\section{Surface Tension and Mass of the Local Soliton}

In this section we define the surface tension for the models discussed in Sect. 2.1.

It is a standard generalization of the surface tension of the (Ising) $)_{2}$ model to scalar and fermion theories in $d=2$ and gauge theories in $d=3$. Let $A=T \times L^{d-1}$ denote the $d$-dimensional lattice centered at the origin, with sides of length $T+1$ in the time direction, and $L+1$ in the space directions. Consider $\partial \Lambda$ as a $d-1$ dimensional lattice and let $\Gamma$ be a curve in the dual lattice $(\partial \Lambda)^{*}$ joining

$$
x_{-}=\left(-\frac{T+1}{2}, \overrightarrow{0}\right) \text { to } x_{+}=\left(\frac{T+1}{2}, \overrightarrow{0}\right) .
$$

The dual of $\Gamma$ in $\partial \Lambda$ is a set of $d-2$ dimensional cells, hence sites in $d=2$ and links in $d=3$; the dual of $\partial \Gamma$ in $\partial \Lambda$ are two $d-1$ dimensional cells in $\partial \Lambda$.

The $\mathscr{Z}$-valued field $\sigma$, introduced in Sect. 2.2, has support on sites in $d=2$ and on links in $d=3$, hence we can define the $q$-boundary conditions, $q \in \mathscr{Z}$, setting $\sigma=0$ on $\partial \Lambda \backslash \Gamma^{*}, \sigma=q$ on $\Gamma^{*}$.

The partition function with $q$-b.c. in the lattice $A=T \times L^{d-1}$ is denoted by $Z_{T \times L^{d-1}}^{(q)}$. Using the hypergauge invariance of the action one can see that $Z^{(q)}$ does not depend on the choice of $\Gamma$.

The surface tension $\tau_{q}$ is defined by

$$
\tau_{q}=\lim _{T \uparrow \infty} \lim _{L \uparrow \infty}-\frac{1}{T} \ln \frac{Z_{T \times L^{d-1}}^{(q)}}{Z_{T \times L^{d-1}}} .
$$

The $q$-b.c. introduce an open line defect in the representation of $Z^{(q)}$ in terms of defects. In fact in the interior of $A, v=d \sigma$, i.e. $d v=0$ and in the boundary

$$
v(\partial \Lambda)(y)=\left\{\begin{array}{rl}
q & y=x_{+} \\
-q & y=x_{-} \\
0 & \text { otherwise }
\end{array},\right.
$$

where the dual is taken considering $v(\partial \Lambda)$ as a field of the lattice $\partial \Lambda$.

Therefore there must exist a connected component of the defect configurations with total charge $q$ joining $x_{-}$to $x_{+}$. In general it will be given by $q$ lines of charge 1 . For $q=1$ the surface tension is the specific free energy of such a defect.

The configurations of defects appearing in $Z^{(q)}$ are therefore quite similar to those appearing in the numerator of the representation (1.7) or (3.2) for the two point disorder correlation $\left\langle s_{1}(x) \Omega, s_{1}(y) \Omega\right\rangle$. This similarity can be made more precise as follows.

Lemma 5.1. Let \langle\rangle$_{T \times L_{d-1}}$ denote the vacuum functional with 0-b.c. in the lattice

$$
\begin{aligned}
\Lambda=T \times L^{d-1} \text { and } x^{+} & =\left(\frac{T}{2}, \overrightarrow{0}\right), x^{-}=\left(-\frac{T}{2}, \overrightarrow{0}\right) . \text { Then } \\
& \left\langle D\left(x^{-},-q, x^{+}, q\right)\right\rangle_{T \times L^{d-1}}=\frac{Z_{T \times L^{d-1}}^{(q)}}{Z_{T \times L^{d-1}}} .
\end{aligned}
$$

Proof. To prove this equality, let $\omega$ be the hypergauge field with support in the dual of a single line of charge $q$ joining $x^{+}$to $x^{-}$, so that $D(\omega)=D\left(x^{-},-q, x^{+}, q\right)$, and 
let $S$ denote a surface in the dual lattice satisfying ( $\Delta \equiv$ symmetric difference):

$$
\partial S=T \Delta \operatorname{supp} \omega^{*}
$$

then apply the transformation $\sigma \rightarrow \sigma+q$ to all the cells dual to $S$ in the modified partition function $Z_{A}(\omega)$ defined in (1.1).

This transformation affects only $(\partial S)^{*}$ and precisely reads $Z(\omega) \rightarrow Z^{(q)}$, i.e. annihilates the $\omega$-field and insert $q$-boundary conditions.

$$
\left\langle D\left(x^{-},-q, x^{+}, q\right)\right\rangle_{T \times L^{d-1}}=\frac{Z_{T \times L^{d-1}}(\omega)}{Z_{T \times L^{d-1}}}=\frac{Z_{T \times L^{d-1}}^{(q)}}{Z_{T \times L^{d-1}}} .
$$

The mass of the soliton $s_{1}(x)$ is defined by

$$
\begin{aligned}
m\left(s_{1}\right) & =\lim _{T \uparrow \infty}-\frac{1}{T}\left\langle D\left(x^{-},-1, x^{+}, 1\right)\right\rangle \\
& =\lim _{T \uparrow \infty}-\frac{1}{T} \lim _{L, T^{\prime} \uparrow \infty} \ln \left\langle D\left(x^{-},-1, x^{+}, 1\right)\right\rangle_{T^{\prime} \times L^{d-1}} .
\end{aligned}
$$

Theorem 5.2. For the models with local soliton sectors to which the C.L.T.H. cluster expansion applies, in the parameter region where the excitation expansion converges, the soliton mass equals the surface tension $\tau_{1}$, i.e.

$$
m\left(s_{1}\right)=\tau_{1} .
$$

Remark 5.3. Since the mass $m\left(s_{1}\right)$ is analytic in the coupling parameters, equality (5.4) extends to the whole domain of analyticity.

Proof of Theorem 5.2. From (5.1) and (5.3) with $\omega$ as above one obtains

$$
m\left(s_{1}\right)-\tau_{1}=\lim _{L, T \uparrow \infty}-\frac{1}{T} \lim _{T^{\prime} \uparrow \infty} \ln \frac{\langle D(\omega)\rangle_{T^{\prime} \times L^{d-1}}}{\langle D(\omega)\rangle_{T \times L^{d-1}}} .
$$

Now we apply the excitation expansion to the numerator and denominator.

However, since the end points of the fluctuating line are fixed, this introduce some constraints in the excitations. More precisely, let $\vec{h}(\varepsilon)$ be a vector going from the point where the excitation $\varepsilon$ attaches to regular cells in the negative time direction, to the point where the excitation $\varepsilon$ attaches to regular cells in the positive time direction.

Then all the sets of excitations $\left\{\varepsilon_{1} \ldots \varepsilon_{n}\right\}$ appearing in the excitation expansion for (5.5) must obey the constraint (see [3]):

$$
\sum_{i=1}^{n} \vec{h}\left(\varepsilon_{i}\right)=\overrightarrow{0}
$$

Therefore, defining $\zeta_{\vec{k}}(\varepsilon)=\zeta(\varepsilon) e^{i \vec{k} \cdot \vec{h}(\varepsilon)}$ we have:

$$
\frac{\langle D(\omega)\rangle_{T^{\prime} \times L^{d-1}}}{\langle D(\omega)\rangle_{T \times L^{d-1}}}=\frac{\frac{1}{(2 \pi)^{d-1} \int d^{d-1} k} \sum_{\left\{\varepsilon_{1} \ldots \varepsilon_{n}\right\} \subset T^{\prime} \times L^{d-1}} \prod_{i=1}^{n} \zeta_{\vec{k}}\left(\varepsilon_{i}\right)}{\frac{1}{(2 \pi)^{d-1}} \int d^{d-1} k \sum_{\left\{\varepsilon_{1} \ldots \varepsilon_{n}\right\} \subset T \times L^{d-1}} \prod_{i=1}^{n} \zeta_{\vec{k}}\left(\varepsilon_{i}\right)} .
$$


We denote by $\boldsymbol{\varepsilon}$ a collection of excitations in which an excitation can occur an arbitrary number of times, and we define the unnormalized measure

$$
d \tilde{\mu}(\vec{h}, \boldsymbol{\varepsilon}) \equiv \frac{d^{d-1} k}{(2 \pi)^{d-1}} \exp \left\{\sum_{\varepsilon \subset T \times L^{d-1}} \frac{\varphi_{T}(\boldsymbol{\varepsilon})}{|\boldsymbol{\varepsilon}| !} \prod_{\varepsilon \in \boldsymbol{\varepsilon}} \zeta_{\vec{k}}(\varepsilon)\right\} .
$$

We denote by $d \mu(\vec{k}, \boldsymbol{\varepsilon})$ the corresponding normalized measure, and by exponentiation we obtain

$$
\frac{\langle D(\omega)\rangle_{T^{\prime} \times L^{d-1}}}{\langle D(\omega)\rangle_{T \times L^{d-1}}}=\int d \mu(\vec{k}, \boldsymbol{\varepsilon}) \exp \left\{\sum_{\substack{\varepsilon \subset T^{\prime} \times L^{d-1} \\ \varepsilon \not T \times L^{d-1}}} \frac{\varphi_{T}(\boldsymbol{\varepsilon})}{|\boldsymbol{\varepsilon}| !} \prod_{\varepsilon \in \boldsymbol{\varepsilon}} \zeta(\varepsilon)\right\} .
$$

The clusters of excitations contributing to the exponential must touch $x^{+}$ and/or $x^{-}$and the plane $t=T$ and/or $t=-T$.

Therefore the exponential is easily bounded uniformly in $L$ by

$$
\left|\sum_{\substack{\boldsymbol{\varepsilon} \subset T^{\prime} \times L^{d-1} \\ \varepsilon \not \subset T \times L^{d-1}}} \frac{\varphi_{T}(\boldsymbol{\varepsilon})}{|\boldsymbol{\varepsilon}| !} \prod_{\varepsilon \in \boldsymbol{\varepsilon}} \zeta_{\vec{k}}(\varepsilon)\right| \leqq K+K e^{-\mu T},
$$

so that

$$
\left|m\left(s_{1}\right)-\tau_{1}\right| \leqq \ln _{L, T \uparrow \infty}-\frac{1}{T} \ln \left(K+K e^{-\mu T}\right)=0 .
$$

\section{Particle Structure Analysis of Stringlike Soliton Sectors}

The construction of the stringlike soliton sectors and the analysis of their particle structure are based essentially on the same methods used in the discussion of the local soliton sectors.

The proof of the existence of the correlation functions $S_{n, m}$ defined in (2.19), is slightly more difficult since explicit cancellations between numerator and denominator are needed in order to show the existence of the thermodynamic limit.

To exhibit the desired cancellations in the correlation functions

$$
\frac{\left\langle D\left(y_{x 1}^{-}, q_{1}, \ldots, \gamma_{y s}^{+}, q_{r+s}\right) \mathcal{O}\left(\Gamma_{1}\right) \ldots \mathcal{O}\left(\Gamma_{m}\right) G_{\partial \Lambda}\right\rangle_{\Lambda}}{\left\langle D\left(\gamma_{x 1}, q_{1}, \ldots, \gamma_{x r}, q_{r}\right) G_{\partial \Lambda}\right\rangle_{\Lambda}^{1 / 2}\left\langle D\left(\gamma_{y 1}, q_{r+1}, \ldots, \gamma_{y s}, q_{r+s}\right) G_{\partial \Lambda}\right\rangle_{\Lambda}^{1 / 2}}
$$

of the model taken as example in Sect. 2.2, we proceed as follows:

1. we integrate out the fermion fields,

2. we apply to the expression obtained for the numerator and the denominator a kind of C.L.H.T. cluster expansion,

3. we (partially) exponentiate the C.L.H.T. cluster expansion by means of an excitation expansion.

Proof of Theorem 2.3. For simplicity we consider expectation values involving a single order variable

$$
\mathcal{O}\left(\Gamma_{x y}\right)=\psi_{x} \prod_{b \in \Gamma_{x y}} e^{\frac{i}{N} A_{b}} \psi_{y}
$$


By integrating out the fermion fields in the numerator of (6.1) we obtain (see e.g. [10]):

$$
\begin{aligned}
\langle D & \left.\left(\gamma_{x 1}^{-}, q_{1}, \ldots, \gamma_{y s}^{+}, q_{r+s}\right) \mathcal{O}\left(\Gamma_{x y}\right) G_{\partial A}\right\rangle_{\Lambda} \\
= & {\left[\sum_{\left\{L_{1}, \ldots, L_{l}, L^{\Gamma}\right\}}\left\langle D\left(\gamma_{x 1}^{-}, q_{1}, \ldots, \gamma_{y s}^{+}, q_{r+s}\right) \prod_{r=1}^{l} \gamma^{L r} \beta_{B}^{|L r|} e^{\frac{i}{N}(A, L r)} \gamma^{L^{\Gamma}} \beta_{B}^{\left|L^{\Gamma}\right|} e^{\frac{i}{N}\left(A, L^{\Gamma}\right)} G_{\partial \Lambda}\right\rangle_{\Lambda}^{0}\right] } \\
& \times\left[\sum_{\left\{L_{1}, \ldots, L_{l}\right\}}\left\langle\prod_{r=1}^{l} \gamma^{L r} \beta_{B}^{|L r|} e^{\frac{i}{N}(A, L r)}\right\rangle_{\Lambda}^{0}\right]^{-1}
\end{aligned}
$$

where: $L_{r}$ denotes a set of linked loops, $L^{\Gamma}$ a line with boundary $\{x, y\}$ and $\left\{L_{1}, \ldots, L_{l}, L^{\Gamma}\right\}$ a set of $L$ 's whose supports are disjoint.

Moreover given a line $L$ we set:

$$
(A, L) \equiv \sum_{b \in L} A_{b}, \quad \gamma^{L} \equiv \operatorname{Tr} \prod_{b \in L} \frac{1+\gamma_{b}}{2} .
$$

Finally \langle\rangle$_{A}^{0}$ denotes the expectation value with respect to the vacuum functional of the non-compact $U(1)$ Higgs model $\left(\beta_{B}=0\right)$ in the lattice $\Lambda$.

Similar formulas hold also for the terms in the denominator. Next we rewrite \langle\rangle$_{A}^{0}$ in terms of the orbit fields and the $v$-field of the Higgs model.

We now apply to (6.2) the C.L.H.T. of Sect. 3.1.

In the expansion for the denominator of (6.2) three types of polymers appear: $v$ and $X$-polymers, as in Sect. 3.1, and $L$-polymers, i.e. the sets of linked loops originated by the integration of the fermion fields (these are still of $X$-type in the terminology of Remark 3.2).

In the expansion for the numerator two other types of polymers appear: the $v^{\omega}$-polymer, as in Sect. 3.1, and the $L^{\Gamma}$ polymer, i.e. the line with boundary $\{x, y\}$, originated by the integration of the fermion fields on $\mathcal{O}\left(\Gamma_{x y}\right)$. A configuration $\left\{v_{1}, \ldots, v_{m}\right\},\left\{X_{1}, \ldots, X_{n}\right\},\left\{L_{1}, \ldots, L_{l}\right\}$ is said to form a cluster on

$$
C=\operatorname{supp}\left(\bigcup_{i=1}^{m} v_{i} \bigcup_{j=1}^{n} X_{j} \bigcup_{r=1}^{l} L_{r}\right)
$$

if

$$
\operatorname{supp}\left(\bigcup_{i} v_{i}^{*} \bigcup_{j} X_{j} \bigcup_{r} S_{r}\right)
$$

is connected for every choice of the surface $\left\{S_{r}: \partial S_{r}=L_{r}\right\}$, i.e. $L_{r}$ is either connected so some $X_{j}$ or linked to some $\left(v_{i}\right)^{*}$.

A configuration $\left\{v_{1}, \ldots, v_{m}, v^{\omega}\right\}\left\{X_{1}, \ldots, X_{n}\right\},\left\{L_{1}, \ldots, L_{l}, L^{\Gamma}\right\}$ is said to form a cluster on

$$
C^{\omega \Gamma}=\operatorname{supp}\left(\bigcup_{i} v_{i} \bigcup_{j} X_{j} \bigcup_{r} v^{\omega} \cup L^{\Gamma}\right)
$$

if every connected component of

$$
\operatorname{supp}\left(\bigcup_{i} v_{i}^{*} \bigcup_{j} X_{j} \bigcup_{r} S_{r} \cup\left(v^{\omega}-\omega\right)^{*} \cup S^{\Gamma}\right)
$$

has non-vanishing intersection with $\operatorname{supp}(d \omega)^{*} \cup \Gamma_{x y}$ for every choice of the surfaces $S_{r}$ and $S^{\Gamma}: \partial S^{\Gamma}=L^{\Gamma} \Delta \Gamma_{x y}$. 
With the same notations of Sect. 3.2b, the activity of a cluster $C^{\omega \Gamma}$ is given by

$$
Z\left(C^{\omega \Gamma}\right)=\sum_{\substack{\left\{v_{1}, \ldots, v_{m}, v^{\omega}\right\}\left\{X_{1}, \ldots, X_{n}\right\}\left\{L_{l}, \ldots, L^{\Gamma}\right\} \\ \text { clusterson } C^{\omega \Gamma}}} Z\left(v_{1}, \ldots, v_{m}, v^{\omega}, X_{1}, \ldots, X_{n}, L_{1}, \ldots, L_{l}, L^{\Gamma}\right)
$$

with

$$
\begin{aligned}
Z\left(v_{1}, \ldots, v_{m}, v^{\omega}, X_{1}, \ldots, X_{n}, L_{1}, \ldots, L_{l}, L^{I}\right) & \\
= & \prod_{i=1}^{m} Z\left(v_{i}\right) Z\left(v_{\omega \omega}\right) \int \prod_{\langle x y\rangle \in C^{\omega \Gamma}} d v\left(\theta_{\langle x y\rangle}\right) \prod_{x \in C^{\omega \Gamma}} d v\left(r_{x}\right) \\
& \cdot \prod_{p \in X} R_{p} \prod_{\langle x y\rangle \in X} R_{\langle x y\rangle} \prod_{r=1}^{l} \gamma^{L r} \beta_{B}^{|L r|} e^{\frac{i}{N}\left(\theta, L_{r}\right)} \\
& \cdot e^{\frac{i}{N}\left(S_{r}, v-\omega\right)} \gamma^{L^{\Gamma}} \beta_{B}^{\left|L^{\Gamma \mid}\right|} e^{\frac{i}{N}\left(\theta, L^{\Gamma} \Delta \Gamma_{x y}\right)} e^{\frac{i}{N}\left(S^{\Gamma}, v-\omega\right)}
\end{aligned}
$$

and a similar definition for $Z(C)$. Assuming the conditions for the coupling constants stated in Theorem 2.3, one can prove, that the C.L.H.T. expansion

$$
(6.2)=\sum_{\left\{\mathbf{C}, C^{\omega \Gamma}\right\}} \frac{\varphi_{T}\left(\mathbf{C}, C^{\omega \Gamma}\right)}{|\mathbf{C}| !} \prod_{C \in \mathbf{C}} Z(C) Z\left(C^{\omega \Gamma}\right)
$$

converges, using the techniques of Sect. 3.

In particular, with the notation of Sect. 2.2 one can prove the bounds,

$$
|Z(C)| \leqq e^{-\left(K_{B}-K_{c}\right)|C|}, \quad\left|Z\left(C^{\omega \Gamma}\right)\right| \leqq e^{-\left(K_{B}-K_{c}\right)|C \omega \Gamma|} \cdot e^{-\left(K_{v}-K_{B}-K_{2}\right)\left|v_{\min }^{\omega}\right|} .
$$

Step 2 is then accomplished by applying the same expansion also to the term in the denominator of (6.1).

To exponentiate the cluster expansions (Step 3) we define the string excitations. A string excitation $\varepsilon^{s}$ relative to $\omega \Gamma$ is given by a set of cells in a configuration $\left\{\mathbf{C}, C^{\omega \Gamma}\right\}$ having maximally connected projection on the time axis and satisfying

$$
\pi\left(\varepsilon_{s}\right) \subset \pi(\omega), \quad \pi\left(\varepsilon_{\mathrm{s}}\right) \cap(\pi(d \omega) \cup \pi(\Gamma))=\emptyset .
$$

Let $\left\{\varepsilon_{i}^{s}\right\}$ be the set of string excitations in $\left\{\mathbf{C}, C^{\omega \Gamma}\right\}$; we define (see Fig. 1):

$$
\begin{gathered}
\mathbf{C}\left(\varepsilon^{s}\right)=\mathbf{C} \cap \varepsilon^{s}, \quad C^{\omega \Gamma}\left(\varepsilon^{s}\right)=C^{\omega \Gamma} \cap \varepsilon^{s}, \\
\dot{C}^{\omega \Gamma}=C^{\omega \Gamma} \backslash\left\{C^{\omega \Gamma}\left(\varepsilon_{i}^{s}\right)\right\}, \quad \dot{\mathbf{C}}=\mathbf{C} \backslash\left\{C\left(\varepsilon_{i}^{s}\right)\right\} .
\end{gathered}
$$

A string excitation $\varepsilon^{s}$ is said to be allowed for $\left\{\dot{\mathbf{C}}, \dot{C}^{\omega \Gamma}\right\}$ iff there exists a configuration $\left\{\mathbf{C}, C^{\omega \Gamma}\right\}$ such that $\varepsilon^{s}$ is a string excitation of this configuration.

Compatibility of excitations is defined as in Sect. 4.

We now outline the idea used to obtain cancellations between numerator and denominator of (6.1) which allows us to take the thermodynamic limit. Let us call $\omega^{1}, \omega^{2}, \omega^{3}$ the hypergauge field respectively in the numerator and in the two-term in the denominator of (6.1), so that

$$
(6.1)=\frac{\left\langle D\left(\omega_{1}\right) \mathcal{O}\left(\Gamma_{x y}\right) G_{\partial \Lambda}\right\rangle_{\Lambda}}{\left\langle D\left(\omega_{2}\right) G_{\partial \Lambda}\right\rangle_{\Lambda}^{1 / 2}\left\langle D\left(\omega_{3}\right) G_{\partial \Lambda}\right\rangle_{\Lambda}^{1 / 2}} .
$$



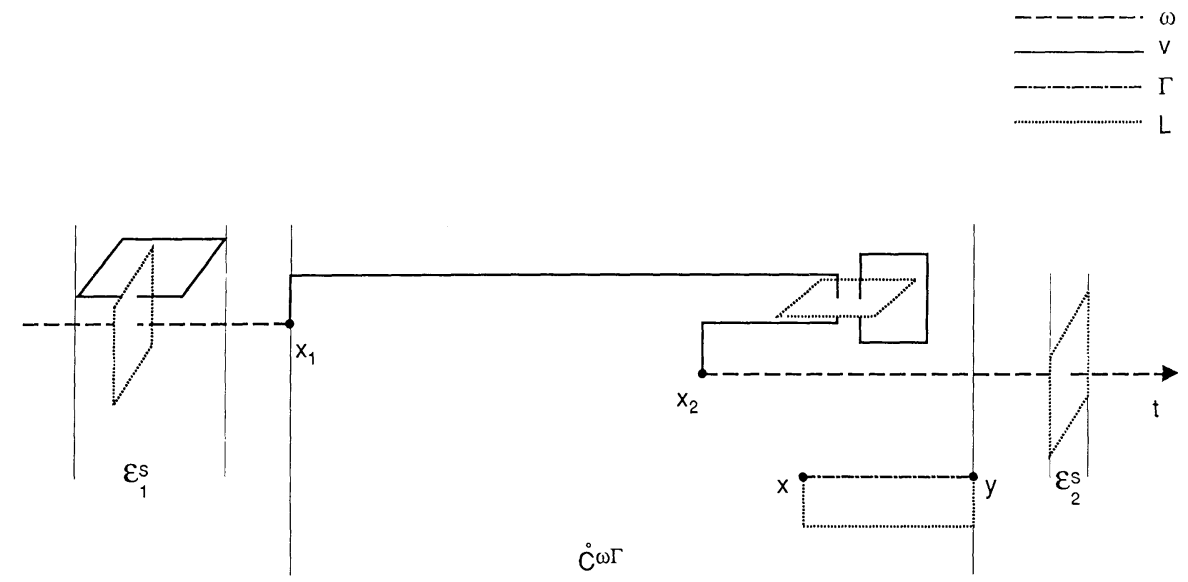

Fig. 1. A configuration $C^{\omega \Gamma}$ with: $\operatorname{supp} d \omega^{*}=\left\{x_{1}, x_{2}\right\}, x_{1}^{0}<0, x_{2}^{0}>0$ and $\Gamma=\Gamma_{x y}$

In the cluster expansions (6.5) for the numerator we first sum over the string excitations compatible, allowed for a fixed $\left\{\dot{\mathbf{C}}, \dot{C}^{\omega_{1} \Gamma}\right\}$ and then sum over all possible $\left\{\dot{\mathbf{C}}, \dot{C}^{\omega_{1} \Gamma}\right\}$. In the denominator, since $\dot{\mathbf{C}}=\emptyset=\dot{C}^{\omega_{2,3}}$, we have only the sum over the string excitations.

In this way both in the numerator and denominator the contributions of the string excitations get exponentiated and the contributions due to the denominator cancel all the contributions in the numerator coming from clusters of excitations which do not touch the clusters $\left\{\dot{\mathbf{C}}, \dot{C}^{\omega_{1} \Gamma}\right\}$. Hence everything is reduced to a cluster expansion in terms of $\left\{\dot{C}, \dot{C}^{\omega_{1} \Gamma}\right\}$ which can be treated as in Sect. 3 .

To implement this idea we first notice the factorization property

$$
\frac{\varphi_{T}\left(\mathbf{C}, C^{\omega T}\right)}{|\mathbf{C}| !}=\prod_{i} \frac{\varphi_{T}\left(\mathbf{C}\left(\varepsilon_{i}^{s}\right), C^{\omega \Gamma}\left(\varepsilon_{i}\right)\right)}{\left|\mathbf{C}\left(\varepsilon_{i}^{s}\right)\right| !} \frac{\varphi_{T}\left(\dot{\mathbf{C}}, \dot{C}^{\omega T}\right)}{|\dot{\mathbf{C}}| !} .
$$

Defining the activity of a string excitation relative to $\omega \Gamma$ by

$$
\zeta^{\omega \Gamma}\left(\varepsilon^{s}\right)=\frac{\left.\varphi_{T}\left(\mathbf{C}\left(\varepsilon^{s}\right)\right), C^{\omega \Gamma}\left(\varepsilon^{s}\right)\right)}{\left|\mathbf{C}\left(\varepsilon^{s}\right)\right| !} \prod_{C \in \mathbf{C}\left(\varepsilon^{s}\right)} Z(C) Z\left(C^{\omega \Gamma}\left(\varepsilon^{s}\right)\right),
$$

and setting

$$
Z^{\omega \Gamma}\left(\varepsilon^{s}\right)=\frac{\varphi_{T}\left(\varepsilon^{s}\right)}{\left|\varepsilon^{s}\right| !} \prod_{\varepsilon^{s} \in \varepsilon^{s}} \zeta^{\omega \Gamma}\left(\varepsilon^{s}\right)
$$

one can rewrite

$$
\begin{aligned}
(6.1)= & {\left[\sum_{\left\{\dot{\mathbf{C}}, \dot{C}^{\left.\omega_{1} \Gamma\right\}}\right.} \frac{\varphi_{T}\left(\dot{\mathbf{C}}, \dot{\boldsymbol{C}}^{\omega_{1} \Gamma}\right)}{|\dot{\mathbf{C}}| !} \prod_{\boldsymbol{C} \in \mathbf{C}} Z(C) Z\left(\dot{C}^{\omega_{1} \Gamma}\right) \exp \left\{\sum_{\boldsymbol{\varepsilon}^{s} \text { allowed for }\left\{\mathbf{C}, C^{\left.\omega_{1} \Gamma\right\}}\right.} Z^{\omega_{1} \Gamma}\left(\boldsymbol{\varepsilon}^{s}\right)\right\}\right] } \\
& \cdot\left[\exp \sum_{\boldsymbol{\varepsilon}^{s}} Z^{\omega_{2}}\left(\boldsymbol{\varepsilon}^{s}\right)\right]^{-1 / 2}\left[\exp \sum_{\boldsymbol{\varepsilon}^{s}} Z^{\omega_{3}}\left(\boldsymbol{\varepsilon}^{s}\right)\right]^{-1 / 2} .
\end{aligned}
$$

We now separate the sum over the string excitations in three terms: excitations $\varepsilon^{s}$ with support completely contained in the positive time lattice, $\Lambda_{+}$, in the negative time lattice, $A_{-}$, intersecting the time zero plane, $\Lambda_{0}$. 
Define

$$
\begin{aligned}
E_{+}^{2}\left(\dot{\mathbf{C}}, \dot{C}^{\omega_{1} \Gamma}\right) & =\left\{\boldsymbol{\varepsilon}^{s} \text { relative to } \omega^{2}: \boldsymbol{\varepsilon}^{s} \subset \Lambda_{+}, \pi\left(\boldsymbol{\varepsilon}^{s}\right) \cap \pi\left(\dot{\mathbf{C}} \cup \dot{C}^{\omega_{1} \Gamma}\right) \neq \emptyset\right\}, \\
E_{-}^{3}\left(\dot{\mathbf{C}}, \dot{C}^{\omega_{1} \Gamma}\right) & =\left\{\boldsymbol{\varepsilon}^{s} \text { relative to } \omega^{3}: \boldsymbol{\varepsilon}^{s} \subset \Lambda_{-}, \pi\left(\boldsymbol{\varepsilon}^{s}\right) \cap \pi\left(\dot{\mathbf{C}} \cup \dot{C}^{\omega_{1} \Gamma}\right) \neq \emptyset\right\}, \\
E_{0}^{2,3} & =\left\{\boldsymbol{\varepsilon}^{s} \text { relative to } \omega^{2,3}: \boldsymbol{\varepsilon}^{s} \cap \Lambda_{0} \neq \emptyset\right\}, \\
E_{+,-}^{1}\left(\dot{\mathbf{C}}, \dot{C}^{\omega_{1} \Gamma}\right) & =\left\{\boldsymbol{\varepsilon}^{s} \text { allowed for }\left\{\dot{\mathbf{C}}, \dot{C}^{\omega_{1} \Gamma}\right\}: \boldsymbol{\varepsilon}^{s} \subset \Lambda_{+,-}\right\}, \\
E_{0}^{1}\left(\dot{\mathbf{C}}, \dot{C}^{\omega_{1} \Gamma}\right) & =\left\{\boldsymbol{\varepsilon}^{s} \text { allowed for }\left\{\dot{\mathbf{C}}, \dot{C}^{\omega_{1} \Gamma}\right\}: \boldsymbol{\varepsilon}^{s} \cap \Lambda_{0} \neq \emptyset\right\} .
\end{aligned}
$$

(For shortness we omit the explicit reference to $\dot{\mathbf{C}}, \dot{C}^{\omega_{1} \Gamma}$, in the following.)

Using also the symmetry for reflection in the time zero plane we have:

$$
\frac{1}{2} \sum_{\varepsilon^{s} \cap \Lambda_{0}=\emptyset} Z^{\omega_{2}}\left(\boldsymbol{\varepsilon}^{s}\right)=\sum_{\boldsymbol{\varepsilon}^{s} \subset \Lambda_{+}} Z^{\omega_{2}}(\boldsymbol{\varepsilon})=\sum_{\boldsymbol{\varepsilon}^{s} \in E_{+}^{1}} Z^{\omega_{1} \Gamma}\left(\boldsymbol{\varepsilon}^{s}\right)+\sum_{\boldsymbol{\varepsilon}^{s} \in E_{+}^{2}} Z^{\omega^{2}}\left(\boldsymbol{\varepsilon}^{s}\right),
$$

and a similar equality holds for $Z^{\omega_{3}}$.

We finally rewrite

$$
\begin{aligned}
(6.1)= & {\left[\sum_{\left\{\mathbf{C}, C^{\omega_{1}} \Gamma\right\}} \frac{\varphi_{T}\left(\dot{\mathbf{C}}, \dot{C}^{\omega_{1}, \Gamma}\right)}{|\dot{\mathbf{C}}| !} \prod_{C \in \dot{\mathbf{C}}} Z(C) Z\left(\dot{C}^{\omega_{1} \Gamma}\right)\right] \exp \left[\sum_{\boldsymbol{\varepsilon}^{s} \in E_{+}^{2}} Z^{\omega_{2}}\left(\boldsymbol{\varepsilon}^{s}\right)+\sum_{\boldsymbol{\varepsilon}^{s} \in E_{-}^{3}} Z^{\omega_{3}}\left(\boldsymbol{\varepsilon}^{s}\right)\right.} \\
& \left.+\sum_{s_{\in} E_{0}^{1}} Z^{\omega_{3}}\left(\boldsymbol{\varepsilon}^{s}\right)-\frac{1}{2} \sum_{\boldsymbol{\varepsilon}^{s} \in E_{0}^{2}} Z^{\omega_{2}}\left(\boldsymbol{\varepsilon}^{s}\right)-\frac{1}{2} \sum_{\boldsymbol{\varepsilon}^{s} \in E_{0}^{3}} Z^{\omega_{3}}\left(\boldsymbol{\varepsilon}^{s}\right)\right] .
\end{aligned}
$$

The theorem is now proved by using the same methods of Sect. 3 together with the bounds:

$$
\begin{gathered}
\sum_{\boldsymbol{\varepsilon} \in E_{0}^{i}}\left|Z^{\omega_{i}}\left(\boldsymbol{\varepsilon}^{s}\right)\right| \leqq K \\
\sum_{\boldsymbol{\varepsilon}^{s} \in E^{i_{+}},-}\left|Z^{\omega_{\imath}}\left(\boldsymbol{\varepsilon}^{s}\right)\right| \leqq e^{-\mathcal{O}\left(K_{B}\right)}\left|\pi\left(\dot{\mathbf{C}} \cup \dot{C}^{\omega_{1} \Gamma}\right)\right| \leqq e^{-\mathcal{O}\left(K_{B}\right)}\left|\dot{\mathbf{C}} \cup \dot{C}^{\omega_{1} \Gamma}\right|,
\end{gathered}
$$

since only clusters of excitations $\boldsymbol{\varepsilon}^{s}$ such that $\pi\left(\boldsymbol{\varepsilon}^{s}\right)$ touches $\pi\left(\Lambda_{0}\right)$ or $\pi\left(\dot{\mathbf{C}} \cup \dot{C}^{\omega_{1} \Gamma}\right)$ contribute to (6.9).

Proof of Theorem 2.4. To prove (2.10), (2.11) we need to analyze in terms of excitations the fluctuating line $\left(v^{\omega}\right)^{*}$ joining 0 to $x$. Our previous expansion was organized in such a way to evidentiate only string excitations, without analyzing the fluctuations of $\left(v^{\omega}\right)^{*}$, hence it has to be slightly modified.

We start from the expansion (6.5):

$$
\begin{aligned}
\left\langle D\left(\gamma_{0}^{-},-1, \gamma_{x}^{+},+1\right) G_{\partial \Lambda}\right\rangle_{\Lambda} & \equiv\left\langle D(\omega) G_{\partial \Lambda}\right\rangle_{\Lambda} \\
& =\sum_{\left\{\mathbf{C}, C_{0 x}\right\}} \frac{\varphi_{T}\left(\mathbf{C}, C_{0 x}\right)}{|\mathbf{C}| !} Z\left(C_{0 x}\right) \prod_{C \in \mathbf{C}} Z(C),
\end{aligned}
$$

where we set $C^{\omega_{1}} \equiv C_{0 x}$.

If we omit from a configuration $\left\{\mathbf{C}, C_{0 x}\right\}$ all regular cells (defined as in Sect. 4) we are left with a set of cells with disjoint projection on the time axis: the excitations. We divide the excitations into two classes: the string excitations, $\varepsilon^{s}$, previously defined, with $\pi\left(\varepsilon^{s}\right) \subset \pi\left(\omega_{1}^{*}\right) \backslash \pi\left(d \omega_{1}^{*}\right)$ and the complementary class of particle excitations denoted by $\varepsilon$ (see Fig. 2). The activity of a particle excitation is denoted by $\zeta(\varepsilon)$ and it is defined as in (4.5); the definition of excitations allowed in $[0, t]$ and the notion of compatibility between excitations are as in Sect. 4 . There is 


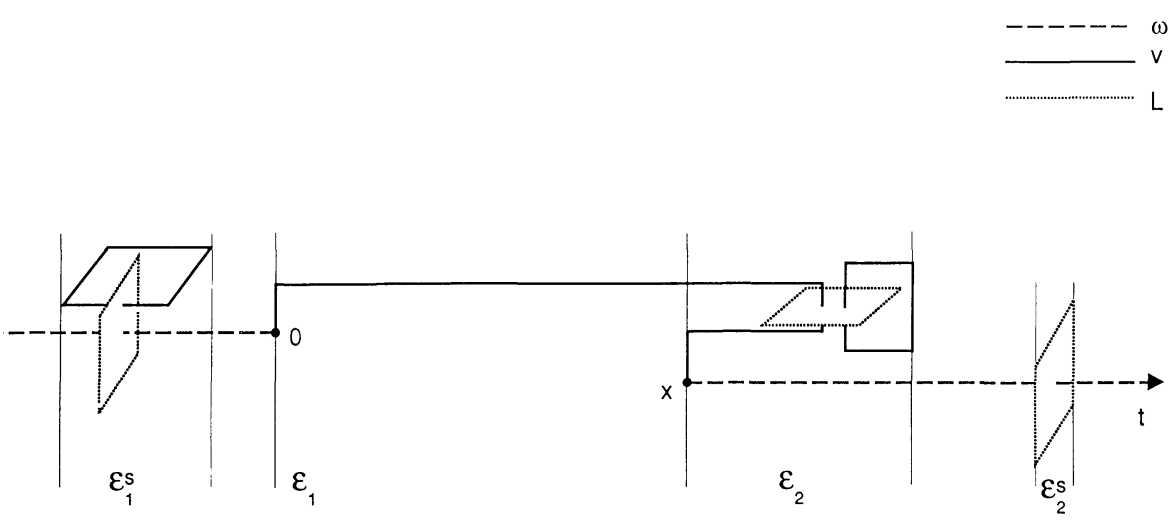

Fig. 2. A configuration $C^{\omega_{1}}$ with $\operatorname{supp} d \omega_{1}^{*}=\{0, x\}$

a one-to-one correspondence between the sets of excitations compatible, allowed in $[0, t]$ and the configurations occurring in the cluster expansion for

$$
\sum_{\vec{x}}\left\langle D\left(\gamma_{0}^{-},-1, \gamma_{x}^{+},+1\right)\right\rangle_{\Lambda}
$$

with $x=(t, \vec{x})$. Using

$$
\begin{aligned}
S\left(\gamma_{0}^{-},-1, \gamma_{x}^{+},+1\right) & =\lim _{\Lambda \uparrow \mathbf{z}_{1 / 2}^{3}} \frac{\left\langle D\left(\gamma_{0}^{-},-1, \gamma_{x}^{+},+1\right) G_{\partial \Lambda}\right\rangle_{\Lambda}}{\left\langle D\left(\gamma_{0}\right) G_{\partial \Lambda}\right\rangle_{\Lambda}^{1 / 2}\left\langle D\left(\gamma_{1}\right) G_{\partial \Lambda}\right\rangle_{\Lambda}^{1 / 2}} \\
& =\lim _{\Lambda \uparrow \mathbf{z}_{1 / 2}^{3}} \frac{\left\langle D\left(\gamma_{0}^{-},-1, \gamma_{x}^{+},+1\right) G_{\partial \Lambda}\right\rangle_{\Lambda}}{\left\langle D\left(\gamma_{0}\right) G_{\partial \Lambda}\right\rangle_{\Lambda}} \\
& \equiv \lim _{\Lambda \uparrow \mathbf{z}_{1 / 2}^{3}} \frac{\left\langle D\left(\omega_{1}\right) G_{\partial \Lambda}\right\rangle_{\Lambda}}{\left\langle D\left(\omega_{2}\right) G_{\partial \Lambda}\right\rangle_{\Lambda}}
\end{aligned}
$$

we obtain

$$
\sum_{\vec{x}} S\left(\gamma_{0}^{-},-1, \gamma_{x}^{+},+1\right)=\lim _{\Lambda \uparrow \mathbf{Z}_{1}^{3}, 2} \frac{\sum_{\left\{\varepsilon_{i}, \varepsilon_{j}^{s}\right\} \subset \Lambda} \prod_{i} \zeta^{\omega_{1}}\left(\varepsilon_{i}\right) \prod_{j} \zeta^{\omega_{1}}\left(\varepsilon_{j}^{s}\right) Z_{\omega}^{t}}{\sum_{\left\{\varepsilon_{j}^{s}\right\} \subset \Lambda} \prod_{j} \zeta^{\omega_{2}}\left(\varepsilon_{j}^{s}\right)}
$$

where $\left\{\varepsilon_{i}, \varepsilon_{j}^{s}\right\}$ in the numerator denotes a set of excitation compatible allowed in $[0, t],\left\{\varepsilon_{j}^{s}\right\}$ in the denominator denotes a set of excitations compatible relative to $\omega_{2}$ and

$$
Z_{\omega} \equiv e^{-\beta_{G} 2 \pi^{2}}
$$

Exponentiating we obtain:

$$
\begin{aligned}
(6.7)= & \lim _{\Lambda \uparrow \mathbf{Z}_{1 / 2}^{3}} Z_{\omega}^{t} \exp \left[\sum_{\left\{\boldsymbol{\varepsilon}, \boldsymbol{\varepsilon}^{s}\right\} \subset \Lambda} \frac{\varphi_{T}\left(\boldsymbol{\varepsilon}, \boldsymbol{\varepsilon}^{s}\right)}{|\boldsymbol{\varepsilon}| !\left|\boldsymbol{\varepsilon}^{s}\right| !} \prod_{\varepsilon \in \boldsymbol{\varepsilon}} \zeta^{\omega_{1}}(\varepsilon) \prod_{e^{s_{\in}} \boldsymbol{\varepsilon}^{s}} \zeta^{\omega_{1}}\left(\varepsilon^{s}\right)\right. \\
& \left.-\sum_{\left\{\boldsymbol{\varepsilon}^{s}\right\} \subset \Lambda} \frac{\varphi_{T}\left(\boldsymbol{\varepsilon}^{s}\right)}{\left|\boldsymbol{\varepsilon}^{s}\right| !} \prod_{\varepsilon^{s} \in \boldsymbol{\varepsilon}^{s}} \zeta^{\omega_{2}}\left(\varepsilon^{s}\right)\right] .
\end{aligned}
$$


All the excitations $\varepsilon^{s}$ satisfying $\pi\left(\varepsilon^{s}\right) \cap[0, t]=\emptyset$ appear both in the numerator and denominator in (6.7) with $\zeta^{\omega_{1}}\left(\varepsilon^{s}\right)=\zeta^{\omega_{2}}\left(\varepsilon^{s}\right)$, therefore all clusters of string excitations $\boldsymbol{\varepsilon}^{s}$ having vanishing projection on $[0, t]$ cancel in the exponential of Eq. (6.8).

The proof of Theorem 2.4 is now an easy consequence of (6.7) and bounds (6.6).

Acknowledgements. I would like to thank J. Fröhlich for many enlightening discussions. Withouth his friendly support this work would never have been completed. The kind hospitality of the E.T.H. in Zürich is also gratefully acknowledged.

\section{References}

1. Fröhlich, J., Marchetti, P.A.: Soliton quantization in lattice field theories. Commun. Math. Phys. 112, 343 (1987) See also Fröhlich, J., Marchetti, P.A.: Europhys. Lett. 2, 933 (1986)

2. Osterwalder, K., Schrader, R.: Axioms for Euclidean Green's functions, I and II. Commun. Math. Phys. 31, 33 (1975), 42, 281 (1975)

Glaser, V.: On the equivalence of the Euclidean and Wightman formulation of field theory. Commun. Math. Phys. 37, 257 (1974)

Fröhlich, J., Osterwalder, K., Seiler, E.: Ann. Math. 118, 461 (1981)

3. Bricmont, J., Fröhlich, J.: Statistical mechanical methods in particle structure analysis of lattice field theories, I and II. Nucl. Phys. B 251 [FS 13], 517 (1985), Commun. Math. Phys. 98, $553(1983)$

4. Glimm, J., Jaffe, A., Spencer, T.: A convergent expansion about mean field theory. I. The expansion. Ann. Phys. 101, 610 (1976)

5. Bellissard, J., Fröhlich, J., Gidas, B.: Soliton mass and surface tension in the $\left(\lambda|\phi|^{4}\right)_{2}$ quantum field model. Commun. Math. Phys. 60, 37 (1978)

6. Fröhlich, J.: Private communication

7. Buchholz, D., Fredenhagen, K.: Locality and the structure of particle states. Commun. Math. Phys. 84, 1 (1982); see also Fredenhagen, K., Marcu, M.: Charged states in $\mathbf{Z}_{2}$ gauge theories. Commun. Math. Phys. 92, 81 (1983)

8. Balaban, T., Brydges, D., Imbrie, J., Jaffe, A.: The mass gap for Higgs models on a unit lattice. Ann. Phys. 158, 281 (1985); see also Ref. [12]

9. Kennedy, T., King, C.: Spontaneous symmetry breakdown in the abelian Higgs model. Commun. Math. Phys. 104, 327 (1986)

10. Seiler, E.: Gauge theories as a problem of constructive quantum field theory and statistical mechanics. Lecture Notes in Physics, Vol. 159. Berlin, Heidelberg, New York: Springer 1982

11. Seiler, E., In: Gauge Theories; fundamental interaction and rigorous results. Proceedings of 1981 Poiava Brasov Summer School. Boston, Birkhäuser 1982

12. Brydges, D., In: Critical phenomean, random systems gauge theories. (Les Houches 1984), Osterwalder et al. (eds.). Amsterdam, New York, Oxford, Tokyo: North-Holland 1986

Communicated by K. Gawedzki

Received November 12, 1987 